\title{
Phantom maps and purity in modular representation theory, I
}

\author{
by
}

\author{
D. J. Benson and G. Ph. G n a c a d j a (Athens, GA)
}

\begin{abstract}
Let $k$ be a field and $G$ a finite group. By analogy with the theory of phantom maps in topology, a map $f: M \rightarrow N$ between $k G$-modules is said to be phantom if its restriction to every finitely generated submodule of $M$ factors through a projective module. We investigate the relationships between the theory of phantom maps, the algebraic theory of purity, and Rickard's idempotent modules. In general, adding one to the pure global dimension of $k G$ gives an upper bound for the number of phantoms we need to compose to get a map which factors through a projective module. However, this bound is not sharp. For example, for the group $\mathbb{Z} / 4 \times \mathbb{Z} / 2$ in characteristic two, the composite of 6 phantom maps always factors through a projective module, whereas the pure global dimension of the group algebra can be arbitrarily large.
\end{abstract}

\section{INTRODUCTION}

A. Allgemeines. In topology, a map $f: X \rightarrow Y$ between CW complexes is said to be a phantom map if its restriction to each skeleton $X^{n}$ is null homotopic. This concept has been around for at least thirty years, and a good survey can be found in McGibbon [25].

As has been observed by Heller [20], there is a strong analogy between maps in topology being null homotopic and maps between modular representations of a finite group factoring through a projective module. This motivated the second author to introduce the notion of phantom map into modular representation theory [16]. A map $f: M \rightarrow N$ between $k G$-modules ( $k$ a field and $G$ a finite group) is said to be phantom if its restriction to every finitely generated submodule of $M$ factors through a projective module. We

1991 Mathematics Subject Classification: Primary 20C20; Secondary 20J06, 55Q05.

The first author is partly supported by a grant from the NSF.

The second author is partly supported by a grant from New Africa Advisers.

The first author gave a talk at the Kazimierz conference on homotopy theory in June 1997, explaining some of the main ideas of this paper. The authors are indebted to Jon Carlson, Dan Christensen and Amnon Neeman for sharing their ideas on the subject. 
write $\operatorname{Ph}_{k G}(M, N)$ for the phantom maps from $M$ to $N$, and $\underline{\mathrm{Ph}}_{k G}(M, N)$ for the quotient of this by the subspace $\mathrm{PHom}_{k G}(M, N)$ consisting of maps which factor through a projective module. In [16], the theory of phantom maps was investigated for countably generated modules. A construction was given there, which produces from any homogeneous regular sequence of length two in the cohomology ring $H^{*}(G, k)$ a pair of countably generated modules $M$ and $N$ with the property that every map between them is phantom, and $\underline{\mathrm{Ph}}_{k G}(M, N)$ has dimension $2^{\aleph_{0}}$. The space of phantom maps was given an interpretation as

$$
\underline{\operatorname{Ph}}_{k G}(M, N) \cong \varlimsup_{\alpha}^{\lim _{\alpha}^{1}} \operatorname{Hom}_{k G}\left(M_{\alpha}, \Omega N\right)
$$

where $M_{\alpha}$ runs over the finitely generated submodules of $M$. Also, as in the topological case, for countably generated $k G$-modules the composite of any two phantom maps factors through a projective module.

Our purpose in this paper is to make a systematic investigation of the extent to which the analogs of known properties of phantom maps in topology hold in the representation theoretic context, without assuming countability.

One of the main tools is the module theoretic notion of purity. In order to make this paper as accessible as possible, we have included some background material from pure homological algebra. This means that the first third of the paper is largely expository. Some of this material can be found in the books of Jensen and Lenzing [22] and Prest [29], the emphasis in both cases being on the connections with model theory; the paper [19] of Gruson and Jensen on the connections with $\varliminf^{i}$; and the monograph [28] of Osofsky for the projective dimension of flat modules.

B. Besonderes. Some of the highlights of this paper are as follows. Sections 2 and 3 are background material on purity, tailored to suit our needs in the rest of the paper. In Section 4.2, we investigate the basic relationship between phantom maps and purity. Christensen [14] has got similar results for phantom maps in the derived category of a ring. The following appear as Proposition 4.2.2, Corollary 4.2.3, Theorem 4.2.4 and Theorem 4.2.5.

Proposition 1.1.1. The following conditions on a map $f: M \rightarrow N$ of $k G$-modules are equivalent:

(i) $f$ is a phantom map.

(ii) The corresponding extension of $\Omega N$ by $M$ (using the isomorphism $\left.\underline{\operatorname{Hom}}_{k G}(M, N) \cong \operatorname{Ext}_{k G}^{1}(M, \Omega N)\right)$ is pure.

(iii) The composite of $f$ with the natural injection $N \rightarrow N^{* *}$ factors through a projective module.

Corollary 1.1.2. Suppose that $M$ and $N$ are $k G$-modules, where $k$ is a field and $G$ is a finite group. If we express $M$ as a filtered colimit $\lim _{\alpha} M_{\alpha}$ 
with $M_{\alpha}$ finitely generated then

$$
\underline{\operatorname{Ph}}_{k G}(M, N) \cong{\underset{\alpha}{\lim }}^{1} \operatorname{Hom}_{k G}\left(M_{\alpha}, \Omega N\right) \cong \operatorname{Pext}_{k G}^{1}(M, \Omega N) .
$$

THEOREM 1.1.3. The following conditions on a $k G$-module $M$ are equivalent:

(i) There are no phantom maps out of $M$.

(ii) $M$ is pure projective.

(iii) $M$ is isomorphic to a direct sum of finitely generated $k G$-modules.

THEOREM 1.1.4. The following conditions on a $k G$-module $N$ are equivalent:

(i) There are no phantom maps into $N$.

(ii) $N$ is pure injective.

(iii) $N$ is isomorphic to a direct summand of a direct product of finitely generated $k G$-modules.

(iv) $N$ is isomorphic to a direct summand of the dual of some module.

(v) The natural injection $N \rightarrow N^{* *}$ splits.

If $N$ satisfies these equivalent conditions, then the endomorphism ring $\operatorname{End}_{k G}(N)$ has the following properties:

(i) The quotient of $\operatorname{End}_{k G}(N)$ by its Jacobson radical $J$ is a von Neumann regular ring.

(ii) Orthogonal idempotent decompositions of the identity element in the quotient ring $\operatorname{End}_{k G}(N) / J$ lift to orthogonal idempotent decompositions of the identity element in $\operatorname{End}_{k G}(N)$.

In particular, if $N$ is indecomposable then $\operatorname{End}_{k G}(N)$ is a (not necessarily commutative) local ring.

The following theorems on the composition of phantom maps appear as Theorems 4.6.1 and 4.6.2.

THeOREM 1.1.5. There is a natural isomorphism between $\underline{\mathrm{Ph}}_{k G}^{n}(M, N)$, the space of maps from $M$ to $N$ which can be written as a composite of $n$ phantom maps, and the image of the natural map

$$
\operatorname{Pext}_{k G}^{n}\left(M, \Omega^{n} N\right) \rightarrow \operatorname{Ext}_{k G}^{n}\left(M, \Omega^{n} N\right) .
$$

THEOREM 1.1.6. Suppose that $|k|=\aleph_{t}$. Then the composite of any $t+2$ phantom maps between $k G$-modules factors through a projective module.

We combine the information coming from the pure global dimension with a method of Jon Carlson to obtain stronger bounds on the number of phantom maps we need to compose in order to be sure of getting a map which factors through a projective module. In particular, it turns out that this number can be strictly smaller than the bound coming from the 
pure global dimension. For example, we prove that for $\mathbb{Z} / 4 \times \mathbb{Z} / 2$ over a field of cardinality $\aleph_{t}$ and characteristic two, the pure global dimension is $t+1$, whereas the composite of six phantom maps always factors through a projective module. This bound is probably not the best possible. Similarly, we prove that for the quaternion group $Q_{8}$ the composite of three phantom maps always factors through a projective module, as does the composite of 18 phantom maps for the semidihedral group $S D_{16}$ and for the Mathieu group $M_{11}$.

In Section 5, we investigate the relationship between the theory of purity and phantom maps, and the theory of Rickard's idempotent modules [30]. We make the following conjecture.

Conjecture 1.1.7. The idempotent $k G$-modules $E(V)$ constructed by Rickard [30] are pure injective.

The information expressed in Theorem 1.1.4 and Conjecture 1.1.7 can be combined with the following statement, which appears in Section 5.2.

THEOREM 1.1.8. The stable endomorphism rings of the idempotent modules $E(\mathcal{V})$ and $F(\mathcal{V})$ are commutative.

It would therefore be a consequence of the conjecture that if $V$ is (projectively) connected then $\operatorname{End}_{k G}(E(V))$ is a commutative local ring. This statement is known to be true by a recent theorem of Daugulis. We prove the conjecture under some restrictive hypotheses in Section 5.3.

Theorem 1.1.9. Suppose that $k$ is an algebraically closed field of characteristic $p$, and that $G$ has p-rank two. If $V$ is a subvariety of $V_{G}$ then $E(V)$ is pure injective. In particular, there are no nontrivial phantom maps from any module to $E(V)$.

We also examine the modules $F(\mathcal{U})$, where $\mathcal{U}$ denotes the collection of all proper nonzero closed homogeneous irreducible subvarieties of $V_{G}$. In particular we prove the following in Section 5.4.

THEOREM 1.1.10. Let $k$ be a field of characteristic $p$ and cardinality $\aleph_{t}$, and let $E$ be an elementary abelian p-group of order $p^{r}$. Then the pure projective dimension of $F(\mathcal{U})$ as a $k E$-module is $\min (r, t+1)$.

We also make the following conjecture.

Conjecture 1.1.11. The module $F(\mathcal{U})$ is pure injective.

We prove this conjecture in the case of a rank two elementary abelian group. The following appears as Theorem 5.4.6.

THEOREM 1.1.12. Let $k$ be a field of characteristic $p$ and $E$ be an elementary abelian p-group of rank two. Then $F(\mathcal{U})$ is a pure injective $k E$-module. 
Finally, in Section 5.5 we investigate the structure of $\underline{\operatorname{End}}_{k G}(E(\mathcal{V}))$. We produce an exact sequence

$$
0 \rightarrow k \rightarrow \underline{\operatorname{End}}_{k G}(E(\mathcal{V})) \rightarrow \underline{\operatorname{Hom}}_{k G}(\Omega F(\mathcal{V}), k) \rightarrow H^{1}(G, k),
$$

which describes the additive structure. The multiplicative structure is shown to be related to Massey products in cohomology. In many cases, it transpires that $\operatorname{End}_{k G}(E(\mathcal{V}))$ has a very large square zero radical, and modulo the radical it is just a copy of the field $k$.

\section{PURITY}

2.1. Pure exact sequences. Let $R$ be a ring. We consider left $R$ modules unless otherwise stated. An exact sequence

$$
\ldots \rightarrow A_{n+1} \stackrel{\alpha_{n+1}}{\longrightarrow} A_{n} \stackrel{\alpha_{n}}{\rightarrow} A_{n-1} \rightarrow \ldots
$$

of $R$-modules is said to be pure exact if for any right $R$-module $M$ the sequence

$$
\ldots \rightarrow M \otimes_{R} A_{n+1} \stackrel{1 \otimes \alpha_{n+1}}{\longrightarrow} M \otimes_{R} A_{n} \stackrel{1 \otimes \alpha_{n}}{\longrightarrow} M \otimes_{R} A_{n-1} \rightarrow \ldots
$$

is exact. This is the same as saying that each exact sequence

$$
0 \rightarrow \operatorname{Ker}\left(\alpha_{n}\right) \rightarrow A_{n} \rightarrow \operatorname{Ker}\left(\alpha_{n-1}\right) \rightarrow 0
$$

is pure exact (i.e., exact after tensoring with any right module).

If

$$
0 \rightarrow A \rightarrow B \rightarrow C \rightarrow 0
$$

is a pure exact sequence, we say that $A \rightarrow B$ is a pure monomorphism and $B \rightarrow C$ is a pure epimorphism. An $R$-submodule $A \subseteq B$ is said to be pure if the corresponding short exact sequence

$$
0 \rightarrow A \rightarrow B \rightarrow B / A \rightarrow 0
$$

is pure exact, i.e., if for every right $R$-module $M$, the map $M \otimes_{R} A \rightarrow M \otimes_{R} B$ is injective.

Lemma 2.1.1. Every $R$-module (resp. right $R$-module) can be expressed as a filtered colimit of finitely presented $R$-modules (resp. right $R$-modules).

Proof. Let $M$ be an $R$-module described by generators $m_{\alpha}, \alpha \in I_{0}$, and relations $r_{\beta}, \beta \in I_{1}$. Each $r_{\beta}$ involves only a finite number of the $m_{\alpha}$. For each finite subset of the generators $I_{0}^{\prime} \subseteq I_{0}$, and for each finite subset of the relations $I_{1}^{\prime} \subseteq I_{1}$ involving only the elements of $I_{0}^{\prime}$, we have a finitely presented module $M_{I_{0}^{\prime}, I_{1}^{\prime}}$ described by the generators in $I_{0}^{\prime}$ and the relations in $I_{1}^{\prime}$.

If $I_{0}^{\prime} \subseteq I_{0}^{\prime \prime}$ and $I_{1}^{\prime} \subseteq I_{1}^{\prime \prime}$ then there is an obvious homomorphism of $R$-modules $M_{I_{0}^{\prime}, I_{1}^{\prime}} \rightarrow M_{I_{0}^{\prime \prime}, I_{1}^{\prime \prime}}$. These form a filtered system of $R$-modules 
and homomorphisms, and it is easy to verify that the colimit is isomorphic to $M$.

The case of right $R$-modules is proved in the same way.

TheOREM 2.1.2. For a short exact sequence of $R$-modules

$$
0 \rightarrow A \rightarrow B \rightarrow C \rightarrow 0
$$

the following conditions are equivalent:

(i) For every right $R$-module $N$ the sequence

$$
0 \rightarrow N \otimes_{R} A \rightarrow N \otimes_{R} B \rightarrow N \otimes_{R} C \rightarrow 0
$$

is exact.

(ii) For every finitely presented right $R$-module $N$ the sequence

$$
0 \rightarrow N \otimes_{R} A \rightarrow N \otimes_{R} B \rightarrow N \otimes_{R} C \rightarrow 0
$$

is exact.

(iii) For every finitely presented $R$-module $M$, the sequence has the lifting property

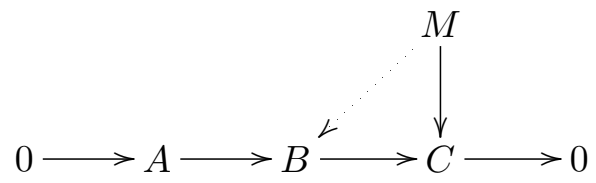

Proof. The equivalence of (i) and (ii) follows from the lemma, together with the fact that a filtered colimit of exact sequences is exact.

To prove that (ii) is equivalent to (iii), we use the notion of the transpose of a finitely presented module. A finite presentation

$$
R^{k} \stackrel{\alpha}{\longrightarrow} R^{n} \rightarrow M \rightarrow 0
$$

of a module $M$ is given by an $n \times k$ matrix $\alpha=\left(\alpha_{i j}\right)$ with entries in $R$. The transpose matrix $\alpha^{T}=\left(\alpha_{j i}\right)$ can be regarded as a presentation of a right $R$-module $N$

$$
D\left(R^{n}\right) \stackrel{\alpha^{T}}{\longrightarrow} D\left(R^{k}\right) \rightarrow N \rightarrow 0,
$$

where $D(-)=\operatorname{Hom}_{R}(-, R)$, and we write $N=\operatorname{Tr}(M)$. Similarly, the transpose of a right module is a left module, and transposing twice gives back the original module.

Given a short exact sequence of $R$-modules

$$
0 \rightarrow A \rightarrow B \rightarrow C \rightarrow 0
$$

and a finite presentation $R^{k} \stackrel{\alpha}{\longrightarrow} R^{n} \rightarrow M \rightarrow 0$, we tensor the sequence with $\alpha^{T}$ and use the fact that for finitely generated free modules $F$,

$$
D(F) \otimes_{R} A \cong \operatorname{Hom}_{R}(F, A),
$$


to deduce that we have a diagram

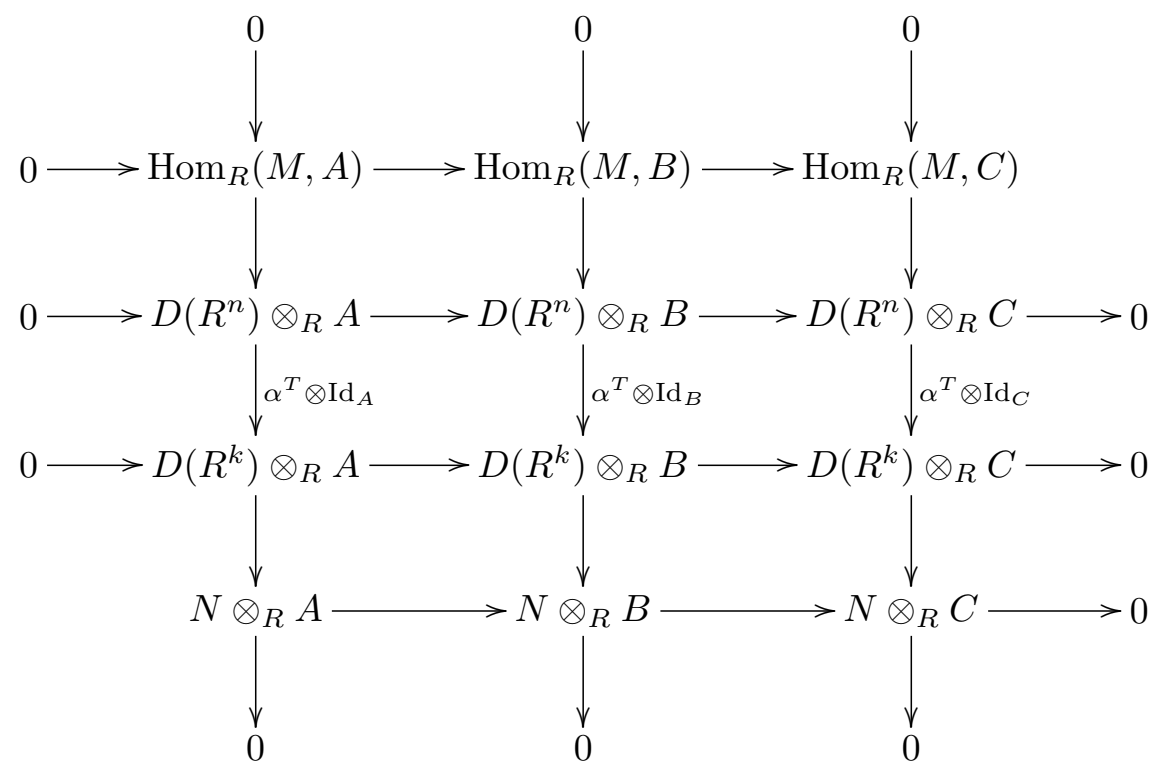

The snake lemma now shows that the bottom sequence is left exact for all finitely presented right modules $N=\operatorname{Tr}(M)$ if and only if the top sequence is right exact for all finitely presented left modules $M$.

\subsection{Pure projective modules}

Definition 2.2.1. An $R$-module $M$ is pure projective if it has the lifting property with respect to pure exact sequences:

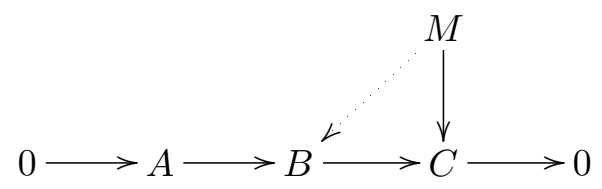

Lemma 2.2.2. Let $M$ be an $R$-module, and using Lemma 2.1.1, write

$$
M=\underset{\alpha}{\lim _{\alpha}} M_{\alpha}
$$

as a filtered colimit of finitely presented modules. Then the obvious surjective map from the direct sum of the $M_{\alpha}$ to $M$ (with kernel $K$, say) gives rise to a pure exact sequence

$$
0 \rightarrow K \rightarrow \bigoplus_{\alpha} M_{\alpha} \rightarrow M \rightarrow 0 .
$$

Proof. Any map from a finitely presented module to $M$ lifts to some $M_{\alpha}$. 
TheOREM 2.2.3. An R-module $M$ is pure projective if and only if it is isomorphic to a direct summand of a (possibly infinite) direct sum of finitely presented $R$-modules.

P r o of. It is clear from the definition that finitely presented modules are pure projective, and that the class of pure projectives is closed under taking arbitrary direct sums and summands.

Conversely, suppose that $M$ is pure projective. Then the pure exact sequence $0 \rightarrow K \rightarrow \bigoplus_{\alpha} M_{\alpha} \rightarrow M \rightarrow 0$ of Lemma 2.2 .2 splits, and so $M$ is isomorphic to a direct summand of $\bigoplus_{\alpha} M_{\alpha}$, and the $M_{\alpha}$ are finitely presented.

TheOREM 2.2.4. Let $R$ be a finite-dimensional algebra over a field. Then the pure projective modules are precisely the direct sums of finitely generated modules.

Proof. This is a consequence of a theorem of Crawley, Jønsson and Warfield (see for example Corollary 26.6 in Anderson and Fuller [1]).

\subsection{Pure injective modules and duality}

Definition 2.3.1. An $R$-module $M$ is pure injective if it has the extension property with respect to pure exact sequences:

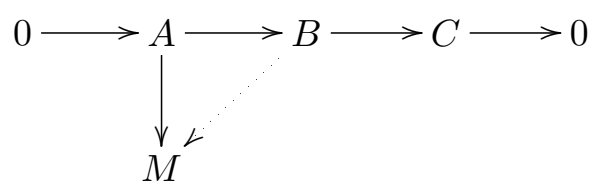

For any right $R$-module $M$, the dual

$$
M^{*}=\operatorname{Hom}_{\mathbb{Z}}(M, \mathbb{Q} / \mathbb{Z})
$$

is a left $R$-module. The action is given by $(r f)(m)=f(m r)$. Similarly, the dual of a left $R$-module $M$ is a right $R$-module $M^{*}$.

Since $\mathbb{Q} / \mathbb{Z}$ is an injective $\mathbb{Z}$-module (i.e., a divisible abelian group), the dual of a short exact sequence of right $R$-modules is a short exact sequence of left $R$-modules and vice versa.

Theorem 2.3.2. For any right $R$-module $M$, the dual $M^{*}$ is a pure injective left $R$-module.

Proof. For any left $R$-module $N$, we have a natural isomorphism

$$
\operatorname{Hom}_{R}\left(N, \operatorname{Hom}_{\mathbb{Z}}(M, \mathbb{Q} / \mathbb{Z})\right) \cong \operatorname{Hom}_{\mathbb{Z}}\left(M \otimes_{R} N, \mathbb{Q} / \mathbb{Z}\right),
$$

which may be written as $\operatorname{Hom}_{R}\left(N, M^{*}\right) \cong\left(M \otimes_{R} N\right)^{*}$.

If $0 \rightarrow A \rightarrow B \rightarrow C \rightarrow 0$ is pure exact then by definition

$$
0 \rightarrow M \otimes_{R} A \rightarrow M \otimes_{R} B \rightarrow M \otimes_{R} C \rightarrow 0
$$


is exact. Dualizing,

$$
0 \rightarrow \operatorname{Hom}_{R}\left(C, M^{*}\right) \rightarrow \operatorname{Hom}_{R}\left(B, M^{*}\right) \rightarrow \operatorname{Hom}_{R}\left(A, M^{*}\right) \rightarrow 0
$$

is exact and so $M^{*}$ is pure injective.

REMARKS 2.3.3. If $R$ is an algebra over a field $k$, it is often better to use the definition

$$
M^{*}=\operatorname{Hom}_{k}(M, k) .
$$

This also takes short exact sequences to short exact sequences because $k$ is an injective $k$-module.

More generally, if $k$ is a commutative ring of coefficients, the appropriate way to dualize is to choose an injective cogenerator $E$ for the category of $k$-modules and set $M^{*}=\operatorname{Hom}_{k}(M, E)$. To say that $E$ is a cogenerator means that given any element $m$ of a $k$-module $M$, there exists a $k$-module homomorphism $f: M \rightarrow E$ such that $f(m) \neq 0$. The point of this definition is to make Lemma 2.4 .1 below work.

For a group $G$ and a commutative coefficient ring $k$, right modules can be regarded as left modules via $g m=m g^{-1}$. Thus the dual of a left module is again a left module.

Corollary 2.3.4. Let $G$ be a group and $k$ a field. Then the dual $M^{*}=$ $\operatorname{Hom}_{k}(M, k)$ of any left $k G$-module $M$ is a pure injective left $k G$-module.

TheOREM 2.3.5. The dual of a pure exact sequence of left (resp. right) $R$-modules

$$
0 \rightarrow A \rightarrow B \rightarrow C \rightarrow 0
$$

is a split exact sequence of right (resp. left) $R$-modules.

Proof. The proof is similar to the proof of the last theorem. We deal with the case of left modules, the other case being similar. Let $M$ be any right $R$-module. Dualizing the statement that

$$
0 \rightarrow M \otimes_{R} A \rightarrow M \otimes_{R} B \rightarrow M \otimes_{R} C \rightarrow 0
$$

is exact, we see that

$$
0 \rightarrow \operatorname{Hom}_{R}\left(M, C^{*}\right) \rightarrow \operatorname{Hom}_{R}\left(M, B^{*}\right) \rightarrow \operatorname{Hom}_{R}\left(M, A^{*}\right) \rightarrow 0
$$

is exact. Applying this with $M=A^{*}$ shows that the identity map on $A^{*}$ lifts to a map $A^{*} \rightarrow B^{*}$, so that the sequence splits.

Pure injective modules have been extensively investigated by logicians, who call them algebraically compact modules. This is because the definition of pure injectivity is equivalent to the following. Given any set of linear equations in the module, with coefficients in the ring, there exists a solution if and only if every finite subset of the equations admits a solution. For more on algebraic compactness, we refer the reader to Chapters 7, 8 and 11 of the 
book of Jensen and Lenzing [22], as well as the book of Prest [29] and the papers of Warfield [32] and Ziegler [34].

We end this section with a method for recognizing pure injective modules. The notation is as follows. If $I$ is an indexing set, we write $M^{(I)}$ for the direct sum of a collection of copies of $M$ indexed by $I$, and $M^{I}$ for the direct product.

Theorem 2.3.6 (Gruson and Jensen). An R-module $M$ is pure injective if and only if for every index set I the natural map from $M^{(I)}$ to $M$ extends to a map from $M^{I}$ to $M$.

P r o of. See Chapter 7 of Jensen and Lenzing [22].

COROLlaRY 2.3.7. An additive functor from one module category to another which preserves direct sums and direct products takes pure injective modules to pure injective modules.

2.4. The double dual. For any $R$-module $M$, there is a natural map

$$
i: M \rightarrow M^{* *}=\operatorname{Hom}_{\mathbb{Z}}\left(\operatorname{Hom}_{\mathbb{Z}}(M, \mathbb{Q} / \mathbb{Z}), \mathbb{Q} / \mathbb{Z}\right)
$$

taking an element $m$ to the map sending $f$ to $f(m)$.

Lemma 2.4.1. The map $i: M \rightarrow M^{* *}$ is injective.

P r o o f. Given any nonzero element $m$ in $M$, there is a nonzero map from the additive subgroup of $M$ generated by $m$ to $\mathbb{Q} / \mathbb{Z}$. Since $\mathbb{Q} / \mathbb{Z}$ is injective, this extends to a map $f$ from $M$ to $\mathbb{Q} / \mathbb{Z}$. Thus the image of $m$ in $M^{* *}$ has a nonzero effect on $f$, and is hence nonzero.

Of course, if $R$ is an algebra over a field $k$ and $M^{*}$ is defined as $\operatorname{Hom}_{k}(M, k)$ then the lemma is even more obvious. More generally, over a commutative ring of coefficients $k$, the definition of injective cogenerator is designed to make the proof of the above lemma work with $M^{*}=$ $\operatorname{Hom}_{k}(M, E)$. We often use the lemma to regard $M$ as a submodule of $M^{* *}$.

TheOrem 2.4.2. The map $i: M \rightarrow M^{* *}$ is a pure monomorphism.

Proof. We must prove that for every right $R$-module $N$, the map $N \otimes_{R} M \rightarrow N \otimes_{R} M^{* *}$ is injective. To see this, we compose with the map

$$
N \otimes_{R} M^{* *} \rightarrow\left(N \otimes_{R} M\right)^{* *}, \quad n \otimes \phi \mapsto(n \otimes-)^{* *}(\phi),
$$

defined as follows. Given a tensor $n \otimes \phi$ in $N \otimes_{R} M^{* *}$, we regard $n \otimes-$ as a map from $M$ to $N \otimes_{R} M$. Then $(n \otimes-)^{* *}$ is a map from $M^{* *}$ to $\left(N \otimes_{R} M\right)^{* *}$ which we can apply to $\phi \in M^{* *}$. It is easy to check that this determines a well defined map.

The composite is the injective map $N \otimes_{R} M \rightarrow\left(N \otimes_{R} M\right)^{* *}$ of Lemma 2.4.1, and so $N \otimes_{R} M \rightarrow N \otimes_{R} M^{* *}$ must be injective. 
Corollary 2.4.3. $M$ is pure injective if and only if the monomorphism $M \rightarrow M^{* *}$ splits.

Proof. If $M$ is pure injective then every pure monomorphism from $M$ splits. Conversely, if $i: M \rightarrow M^{* *}$ splits then $M$ is a direct summand of a pure injective module, and is hence pure injective.

COROllary 2.4.4. An R-module is pure injective if and only if it is isomorphic to a direct summand of a dual of some right $R$-module.

P r o of. This follows from Corollary 2.4.3 together with Theorem 2.3.2.

Corollary 2.4.5. Let $R$ be a finite-dimensional algebra over a field. Then an R-module is pure injective if and only if it is isomorphic to a direct summand of a (possibly infinite) direct product of finite-dimensional $R$-modules.

Proof. Since finite-dimensional modules are isomorphic to their own double duals, a direct product $\prod_{\alpha} M_{\alpha}$ of finite-dimensional $R$-modules is isomorphic to the dual of $\bigoplus_{\alpha} M_{\alpha}^{*}$, and so any summand of such a direct product is pure injective by Corollary 2.4.4.

Conversely, if $M$ is any right $R$-module, writing $M=\lim _{\alpha} M_{\alpha}$ as a filtered colimit of finite-dimensional right modules, by Lemma 2.2 .2 we have a pure exact sequence

$$
0 \rightarrow K \rightarrow \bigoplus_{\alpha} M_{\alpha} \rightarrow M \rightarrow 0 .
$$

By Theorem 2.3.5, the dual sequence

$$
0 \rightarrow M^{*} \rightarrow \prod_{\alpha} M_{\alpha}^{*} \rightarrow K^{*} \rightarrow 0
$$

of left $R$-modules splits, and so $M^{*}$ is a direct summand of a direct product of finite-dimensional $R$-modules. It now follows from Corollary 2.4.4 that every pure injective $R$-module is a direct summand of a direct product of finite-dimensional modules.

2.5. Pure homological algebra. The development of pure homological algebra exactly parallels that of relative homological algebra.

Definition 2.5.1. A pure projective resolution of an $R$-module $M$ is a pure exact sequence

$$
\ldots \rightarrow Q_{2} \rightarrow Q_{1} \rightarrow Q_{0} \rightarrow M \rightarrow 0
$$

in which the modules $Q_{n}(n \geq 0)$ are pure projective.

LEMmA 2.5.2. Every $R$-module $M$ has a pure projective resolution.

Pr o of. This follows immediately from Lemma 2.2.2. 
Definition 2.5.3. A pure injective resolution of an $R$-module $N$ is a pure exact sequence

$$
0 \rightarrow N \rightarrow J_{0} \rightarrow J_{1} \rightarrow \ldots
$$

in which the modules $J_{n}(n \geq 0)$ are pure injective.

Lemma 2.5.4. Every $R$-module $N$ has a pure injective resolution.

Proof. This follows from Theorems 2.3.2 and 2.4.2.

Theorem 2.5.5 (Comparison theorem). (i) Any map $f: M \rightarrow M^{\prime}$ of $R$-modules lifts to a map of pure projective resolutions

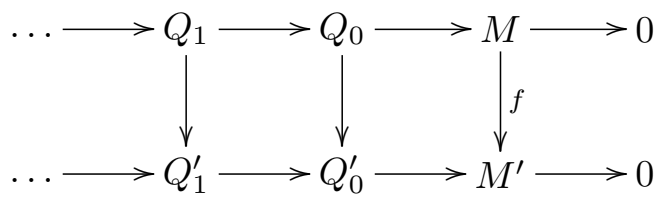

Any two such lifts are chain homotopic.

(ii) Any map $g: N \rightarrow N^{\prime}$ extends to a map of pure injective resolutions

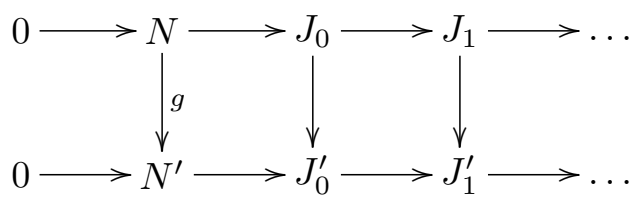

Any two such lifts are chain homotopic.

Definition 2.5.6. We write $\operatorname{Pext}_{R}^{i}(M, N)$ for the cohomology of the complex

$$
0 \rightarrow \operatorname{Hom}_{R}\left(Q_{0}, N\right) \rightarrow \operatorname{Hom}_{R}\left(Q_{1}, N\right) \rightarrow \operatorname{Hom}_{R}\left(Q_{2}, N\right) \rightarrow \ldots
$$

where $\ldots \rightarrow Q_{2} \rightarrow Q_{1} \rightarrow Q_{0} \rightarrow M \rightarrow 0$ is a pure projective resolution of $M$.

This is well defined up to canonical isomorphism (by the comparison theorem), and functorial in both variables. Given a pure exact sequence

$$
0 \rightarrow M_{1} \rightarrow M_{2} \rightarrow M_{3} \rightarrow 0
$$

there is an induced long exact sequence

$$
\begin{aligned}
& 0 \rightarrow \operatorname{Hom}_{R}\left(M_{3}, N\right) \rightarrow \operatorname{Hom}_{R}\left(M_{2}, N\right) \rightarrow \operatorname{Hom}_{R}\left(M_{1}, N\right) \rightarrow \\
& \operatorname{Pext}_{R}^{1}\left(M_{3}, N\right) \rightarrow \operatorname{Pext}_{R}^{1}\left(M_{2}, N\right) \rightarrow \operatorname{Pext}_{R}^{1}\left(M_{1}, N\right) \rightarrow \operatorname{Pext}_{R}^{2}\left(M_{3}, N\right) \rightarrow \ldots
\end{aligned}
$$

Given a pure exact sequence

$$
0 \rightarrow N_{1} \rightarrow N_{2} \rightarrow N_{3} \rightarrow 0
$$

there is an induced long exact sequence

$$
\begin{aligned}
0 & \rightarrow \operatorname{Hom}_{R}\left(M, N_{1}\right) \rightarrow \operatorname{Hom}_{R}\left(M, N_{2}\right) \rightarrow \operatorname{Hom}_{R}\left(M, N_{3}\right) \rightarrow \\
& \operatorname{Pext}_{R}^{1}\left(M, N_{1}\right) \rightarrow \operatorname{Pext}_{R}^{1}\left(M, N_{2}\right) \rightarrow \operatorname{Pext}_{R}^{1}\left(M, N_{3}\right) \rightarrow \operatorname{Pext}_{R}^{2}\left(M, N_{1}\right) \rightarrow \ldots
\end{aligned}
$$


Proposition 2.5.7. $\operatorname{Pext}^{i}(M, N)$ is naturally isomorphic to the cohomology of the complex

$$
0 \rightarrow \operatorname{Hom}_{R}\left(M, J_{0}\right) \rightarrow \operatorname{Hom}_{R}\left(M, J_{1}\right) \rightarrow \operatorname{Hom}_{R}\left(M, J_{2}\right) \rightarrow \ldots
$$

where $0 \rightarrow N \rightarrow J_{0} \rightarrow J_{1} \rightarrow J_{2} \rightarrow \ldots$ is a pure injective resolution of $N$.

Proof. The proof follows exactly the argument in the case of ordinary homological algebra (see for example Proposition 2.5.5 of [5]).

Definition 2.5.8. The pure projective dimension of an $R$-module $M$ is the smallest length $d$ of a pure projective resolution

$$
0 \rightarrow Q_{d} \rightarrow \ldots \rightarrow Q_{0} \rightarrow M \rightarrow 0,
$$

or infinity if no such resolution of finite length exists. Equivalently, it is the smallest value of $d$ such that $\operatorname{Pext}_{R}^{i}(M, N)=0$ for all $R$-modules $N$ and all integers $i>d$.

The pure injective dimension of an $R$-module $N$ is the smallest length $d$ of a pure injective resolution

$$
0 \rightarrow N \rightarrow J_{0} \rightarrow \ldots \rightarrow J_{d} \rightarrow 0,
$$

or infinity if no such resolution of finite length exists. Equivalently, it is the smallest value of $d$ such that $\operatorname{Pext}_{R}^{i}(M, N)=0$ for all $R$-modules $M$ and all integers $i>d$.

The pure global dimension of $R$ is the maximum of the pure projective dimensions of $R$-modules (or infinity if there is no upper bound). This is the same as the maximum of the pure injective dimensions of $R$-modules.

2.6. Cardinality and $\lim ^{i}$. If $M$ is finitely presented then it has pure projective dimension zero. If $M$ is countably presented, then it can be written as a colimit $M=\varliminf_{\longrightarrow} M_{n}$ of a sequence

$$
M_{0} \stackrel{s}{\rightarrow} M_{1} \stackrel{s}{\rightarrow} \ldots
$$

of finitely presented modules. It follows that there is a short exact sequence

$$
0 \rightarrow \bigoplus_{n} M_{n} \stackrel{1-s}{\longrightarrow} \bigoplus_{n} M_{n} \rightarrow M \rightarrow 0 .
$$

It is easy to check that this sequence is pure exact, and is hence a pure projective resolution of $M$. It follows that $M$ has pure projective dimension at most one.

More generally, for any $\aleph$-presented $R$-module $M$ ( $\aleph$ a cardinal), we can write $M$ as a filtered colimit $\underline{\lim } M_{\alpha}$ where $\alpha$ runs over a filter of cardinality $\aleph$ and each $M_{\alpha}$ is finitely presented. We write $f_{\alpha, \beta}$ for the maps $M_{\alpha} \rightarrow M_{\beta}$ in this filtered system. We form an exact sequence of filtered systems (indexed 
by $\alpha$ ) as follows:

$$
\begin{aligned}
& \cdots \rightarrow\left\{\bigoplus_{\alpha_{0} \leq \alpha_{1} \leq \alpha_{2} \leq \alpha} M_{\alpha_{0}, \alpha_{1}, \alpha_{2}, \alpha}\right\} \\
& \stackrel{\partial_{2}}{\longrightarrow}\left\{\bigoplus_{\alpha_{0} \leq \alpha_{1} \leq \alpha} M_{\alpha_{0}, \alpha_{1}, \alpha}\right\} \stackrel{\partial_{1}}{\longrightarrow}\left\{\bigoplus_{\alpha_{0} \leq \alpha} M_{\alpha_{0}, \alpha}\right\} \stackrel{\partial_{0}}{\longrightarrow}\left\{M_{\alpha}\right\} \rightarrow 0
\end{aligned}
$$

where $M_{\alpha_{0}, \ldots, \alpha_{n}, \alpha}$ is a copy of $M_{\alpha_{0}}$. The map $\partial_{n}$ is $\sum_{i=0}^{n}(-1)^{i} j_{i}$, where $j_{i}$ is defined on $M_{\alpha_{0}, \ldots, \alpha_{n}, \alpha}$ to take it via the "identity map" to $M_{\alpha_{0}, \ldots, \widehat{\alpha}_{i}, \ldots, \alpha_{n}, \alpha}$ (the hat here denotes that the $i$ th subscript is missing) if $i>0$ and via $f_{\alpha_{0}, \alpha_{1}}$ if $i=0$. The map $\partial_{0}$ is defined on $M_{\alpha_{0}, \alpha}$ as $f_{\alpha_{0}, \alpha}$.

To see that the sequence is pure exact, one can verify that it is a complex (i.e. $\partial_{n} \partial_{n+1}=0$ for every $n \geq 0$ ) which has a contracting homotopy by $R$-homomorphisms. Such a contracting homotopy $\ldots, s_{2}, s_{1}, s_{0}, s_{-1}$ is as follows: $s_{n}$ maps $M_{\alpha_{0}, \ldots, \alpha_{n}, \alpha}$ into $M_{\alpha_{0}, \ldots, \alpha_{n}, \alpha, \alpha}$ via $(-1)^{n+1} \mathrm{Id}$.

Taking colimits, we obtain

$$
\cdots \rightarrow \bigoplus_{\alpha_{0} \leq \alpha_{1} \leq \alpha_{2}} M_{\alpha_{0}, \alpha_{1}, \alpha_{2}} \stackrel{\partial_{2}}{\longrightarrow} \bigoplus_{\alpha_{0} \leq \alpha_{1}} M_{\alpha_{0}, \alpha_{1}} \stackrel{\partial_{1}}{\longrightarrow} \bigoplus_{\alpha_{0}} M_{\alpha_{0}} \stackrel{\partial_{0}}{\longrightarrow} M \rightarrow 0,
$$

which is a pure projective resolution of $M$. If $N$ is another $R$-module, we can take homomorphisms

$$
\begin{aligned}
0 \rightarrow\left\{\operatorname{Hom}_{R}\left(M_{\alpha}, N\right)\right\} & \stackrel{\partial_{0}^{*}}{\longrightarrow}\left\{\prod_{\alpha_{0} \leq \alpha} \operatorname{Hom}_{R}\left(M_{\alpha_{0}, \alpha}, N\right)\right\} \\
\stackrel{\partial_{1}^{*}}{\longrightarrow} & \left\{\prod_{\alpha_{0} \leq \alpha_{1} \leq \alpha} \operatorname{Hom}_{R}\left(M_{\alpha_{0}, \alpha_{1}, \alpha}, N\right)\right\} \\
& \stackrel{\partial_{2}^{*}}{\longrightarrow}\left\{\prod_{\alpha_{0} \leq \alpha_{1} \leq \alpha_{2} \leq \alpha} \operatorname{Hom}_{R}\left(M_{\alpha_{0}, \alpha_{1}, \alpha_{2}, \alpha}, N\right)\right\} \rightarrow \ldots
\end{aligned}
$$

This is the standard resolution of the inverse filtered system $\left\{\operatorname{Hom}_{R}\left(M_{\alpha}, N\right)\right\}$ for the purpose of calculating the higher derived functors of $\varliminf_{\longleftarrow}$. So applying $\underset{l i m}{\longleftarrow}$ to this sequence, the cohomology of the complex

$$
\begin{aligned}
0 \rightarrow & \operatorname{Hom}_{R}(M, N) \stackrel{\partial_{0}^{*}}{\longrightarrow} \prod_{\alpha_{0}} \operatorname{Hom}_{R}\left(M_{\alpha_{0}}, N\right) \\
& \stackrel{\partial_{1}^{*}}{\longrightarrow} \prod_{\alpha_{0} \leq \alpha_{1}} \operatorname{Hom}_{R}\left(M_{\alpha_{0}, \alpha_{1}}, N\right) \stackrel{\partial_{2}^{*}}{\longrightarrow} \prod_{\alpha_{0} \leq \alpha_{1} \leq \alpha_{2}} \operatorname{Hom}_{R}\left(M_{\alpha_{0}, \alpha_{1}, \alpha_{2}}, N\right) \rightarrow \ldots
\end{aligned}
$$

calculates both $\varliminf_{\alpha}^{i} \operatorname{Hom}_{R}\left(M_{\alpha}, N\right)$ and $\operatorname{Pext}_{R}^{i}(M, N)$. Thus we have

THEOREM 2.6.1. If the R-module $M$ is written as a filtered colimit of finitely presented modules

$$
M=\underset{\alpha}{\lim } M_{\alpha},
$$


then there are natural isomorphisms

$$
\operatorname{Pext}_{R}^{i}(M, N) \cong{\underset{\alpha}{\alpha}}_{\lim ^{i}} \operatorname{Hom}_{R}\left(M_{\alpha}, N\right) .
$$

Now Roos [31] has shown that if a filter has cardinality at most $\aleph_{t}$ then the corresponding functors $\lim ^{i}$ vanish for $i>t+1$. So we have the following corollary.

COROLlaRY 2.6.2. If $M$ is $\aleph_{t}$-presented, then $M$ has pure projective dimension at most $t+1$.

Gruson and Jensen $[18,19]$ have proved the following:

THEOREM 2.6.3. If a ring $R$ has cardinality $\aleph_{t}$ then $R$ has pure global dimension at most $t+1$.

2.7. Flat modules. Tensoring with any module is right exact. An $R$ module $M$ is said to be flat if tensoring with $M$ is also left exact; in other words if $\operatorname{Tor}_{1}^{R}(N, M)=0$ for all right $R$-modules $N$. It follows from the long exact sequence for Tor that any short exact sequence ending with a flat module is pure exact. If

$$
0 \rightarrow M^{\prime} \rightarrow P \rightarrow M \rightarrow 0
$$

is a short exact sequence with $M$ flat and $P$ projective, then the long exact sequence for Tor shows that $M^{\prime}$ is also flat. So a projective resolution of a flat module is also a pure projective resolution. So we have the following.

Proposition 2.7.1. If $M$ is flat then for any $R$-module $N$,

$$
\operatorname{Pext}_{R}^{i}(M, N) \cong \operatorname{Ext}_{R}^{i}(M, N) .
$$

In particular, the pure projective dimension and projective dimension of $M$ coincide.

Corollary 2.7.2. If $M$ is finitely presented and flat then $M$ is projective.

Proof. This follows from the proposition, using the fact that finitely presented modules are pure projective.

COROLlary 2.7.3. If $M$ is $\aleph_{t}$-presented and flat then $M$ has projective dimension at most $t+1$.

Pro of. This follows from Corollary 2.6.2 and Proposition 2.7.1.

A good example is the field of fractions of a polynomial ring. Let $k$ be a field of cardinality $\aleph_{t}$ and let $k\left[x_{1}, \ldots, x_{r}\right]$ be a polynomial ring over $k$ in $r$ variables. Then every module over $k\left[x_{1}, \ldots, x_{r}\right]$ has projective dimension at most $r$ (because of the Koszul complex) and pure projective dimension 
at most $t+1$ (by Theorem 2.6.3). Osofsky ([28, Corollary 2.59] and [27, Theorem 6.4]) has proved the following:

THEOREM 2.7.4. Let $k$ be a field of cardinality $\aleph_{t}$. Then the flat module $k\left(x_{1}, \ldots, x_{r}\right)$ over the polynomial ring $k\left[x_{1}, \ldots, x_{r}\right]$ has projective dimension $\min (r, t+1)$. The graded ring of fractions $k\left(x_{1}, \ldots, x_{r}\right)_{\mathrm{gr}}$ (in which we only invert nonzero homogeneous elements) has the same projective dimension $\min (r, t+1)$.

\section{FUNCTOR CATEGORIES}

\subsection{Grothendieck categories}

Definition 3.1.1. A Grothendieck category $\mathcal{C}$ is an abelian category satisfying

(i) $\mathcal{C}$ has a set $X$ of generators. This means that every object in $\mathcal{C}$ is an epimorphic image of a coproduct of objects in $X$.

(ii) (Grothendieck's axiom AB5) All small colimits exist, and filtered colimits are exact.

For example, the category of $R$-modules is a Grothendieck category with ${ }_{R} R$ as a generator.

If $\mathcal{C}$ is a small additive category, then the category $\operatorname{Fun}(\mathcal{C}, A b)$ of additive covariant functors from $\mathcal{C}$ to abelian groups is a Grothendieck category. By Yoneda's lemma, the representable functors $\operatorname{Hom}_{\mathcal{C}}(M,-)$ are projective objects. Given any functor $F$ in $\operatorname{Fun}(\mathcal{C}, A b)$, Yoneda's lemma gives us a surjective natural transformation

$$
\bigoplus_{M, x \in F(M)} \operatorname{Hom}_{\mathcal{C}}(M,-) \rightarrow F
$$

where for $M$ an object in $\mathcal{C}$ and $x \in F(M)$, the natural transformation $\operatorname{Hom}_{\mathcal{C}}(M,-) \rightarrow F$ is the one corresponding to $x$ under the Yoneda equivalence. So the functors $\operatorname{Hom}_{\mathcal{C}}(M,-)$ form a set of projective generators. Small colimits exist because $\lim F_{\alpha}$ can be taken to be the functor assigning to an object $M$ of $\mathcal{C}$ the abelian group $\lim F_{\alpha}(M)$. Since filtered colimits of abelian groups are exact, the same is true in $\operatorname{Fun}(\mathcal{C}, A b)$.

In any abelian category, we can talk about subobjects of an object to mean equivalence classes of monomorphisms into that object. Containment between one subobject and another also has an obvious meaning. We can talk about the intersection of two subobjects $A$ and $B$ of an object $M$ as the pullback of the corresponding monomorphisms (since a pullback can be expressed as a kernel, abelian categories have pullbacks). There is a natural monomorphism from the pushout $A+B$ of $A$ and $B$ along $A \cap B$ to $M$, and so the sum $A+B$ can be viewed as a subobject of $M$ : 


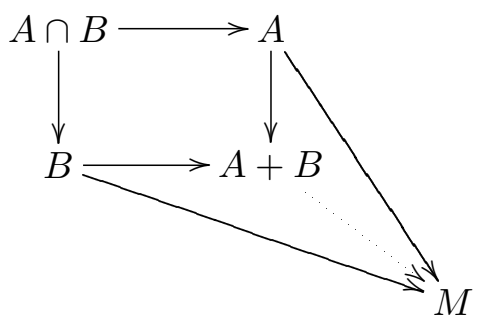

If $A \cap B=0$ then the pushout is isomorphic to the direct sum $A \oplus B$, and in this case we say that $A+B$ is an internal direct sum in $M$. Existence and exactness of filtered colimits has the effect that we can talk about the union of an arbitrary directed collection of subobjects of an object. This allows us to apply Zorn's lemma to obtain maximal subobjects satisfying suitable conditions. In particular, the usual proof of the existence of injective envelopes in a module category works just as well in a Grothendieck category.

3.2. Pure injectives as injective functors. We write $\operatorname{Mod}(R)$ for the category of $R$-modules and module homomorphisms, and we write $\bmod (R)$ for the full subcategory consisting of finitely presented modules.

THEOREM 3.2.1. The functor

$$
\operatorname{Mod}(R) \rightarrow \operatorname{Fun}\left(\bmod \left(R^{\mathrm{op}}\right), \mathrm{Ab}\right), \quad M \mapsto-\otimes_{R} M,
$$

is a full embedding, which preserves direct sums and direct products.

A functor $F$ in $\operatorname{Fun}\left(\bmod \left(R^{\mathrm{op}}\right), \mathrm{Ab}\right)$ is injective if and only if it is isomorphic to $-\otimes_{R} M$ for some pure injective module $M$. The module $M$ is given by $M=F\left(R_{R}\right)$.

P r o of. The module $M$ can be recovered from the corresponding functor, as $R \otimes_{R} M$. Furthermore, a natural transformation from $-\otimes_{R} M$ to $-\otimes_{R} M^{\prime}$ is determined on finitely presented modules by its effect on $R$.

Preservation of direct sums is clear. For direct products, let

$$
R^{k} \rightarrow R^{n} \rightarrow N \rightarrow 0
$$

be a presentation of a finitely presented module $N$, and let $M_{\alpha}$ be a collection of $R$-modules. Since tensor product is right exact, we have a commutative diagram

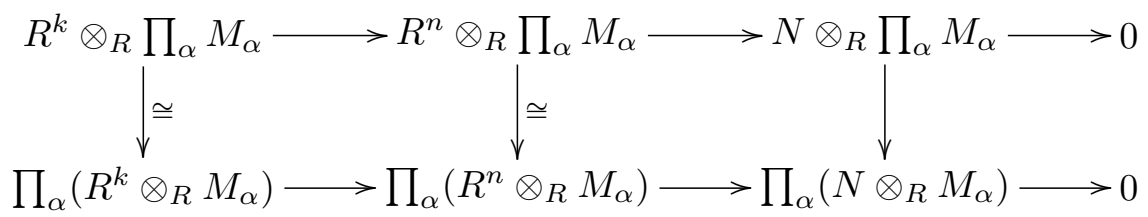

This proves that direct products are preserved. 
If $F: \bmod \left(R^{\mathrm{op}}\right) \rightarrow \mathrm{Ab}$ is injective, we first prove that $F$ is right exact. If $A \rightarrow B \rightarrow C \rightarrow 0$ is exact in $\bmod \left(R^{\mathrm{op}}\right)$ then we can define a functor $X$ via

$$
0 \rightarrow \operatorname{Hom}_{R}(C,-) \rightarrow \operatorname{Hom}_{R}(B,-) \rightarrow \operatorname{Hom}_{R}(A,-) \rightarrow X \rightarrow 0 .
$$

Since $F$ is injective, taking natural transformations to $F$ is exact. Using Yoneda's lemma, we get an exact sequence

$$
0 \rightarrow \operatorname{Nat}(X, F) \rightarrow F(A) \rightarrow F(B) \rightarrow F(C) \rightarrow 0 .
$$

It follows that $F$ is right exact.

Since $R$ is an $R$-R-bimodule via left and right multiplication, $F\left(R_{R}\right)$ is a left $R$-module. If $N$ is a finitely presented module, we have a natural map

$$
N \otimes_{R} F\left(R_{R}\right) \rightarrow F(N), \quad n \otimes x \mapsto F\left(\varrho_{n}\right)(x),
$$

where $\varrho_{a}: R_{R} \rightarrow A$ is defined by $\varrho_{a}(r)=a r$. This is an isomorphism for $N$ a finitely generated free module. More generally, if $R^{k} \rightarrow R^{n} \rightarrow N \rightarrow 0$ is a presentation of a finitely presented module $N$, then since both tensor product and $F$ are right exact, we have a commutative diagram

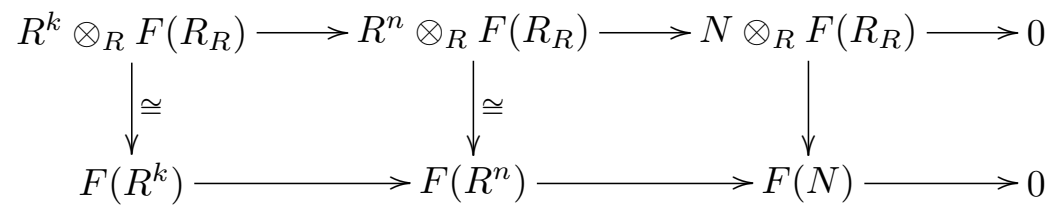

which proves that $F$ is isomorphic to $-\otimes_{R} F\left(R_{R}\right)$.

To see that $M=F\left(R_{R}\right)$ is pure injective, suppose that $0 \rightarrow M \rightarrow M^{\prime} \rightarrow$ $M^{\prime \prime} \rightarrow 0$ is a pure exact sequence of left $R$-modules. Then

$$
0 \rightarrow-\otimes_{R} M \rightarrow-\otimes_{R} M^{\prime} \rightarrow-\otimes_{R} M^{\prime \prime} \rightarrow 0
$$

is a short exact sequence in $\operatorname{Fun}\left(\bmod \left(R^{\mathrm{op}}\right), \mathrm{Ab}\right)$. Since $-\otimes_{R} M$ is injective, this splits. Since the embedding of $\operatorname{Mod}(R)$ in $\operatorname{Fun}\left(\bmod \left(R^{\mathrm{op}}\right), \mathrm{Ab}\right)$ is full, this implies that the embedding of $M$ in $M^{\prime}$ also splits.

Conversely, if $M$ is pure injective, embed $-\otimes_{R} M$ into an injective functor $F$. Then $F$ is of the form $-\otimes_{R} N$ for some pure injective module $N$. Since the embedding of $\operatorname{Mod}(R)$ in $\operatorname{Fun}\left(\bmod \left(R^{\text {op }}\right), \mathrm{Ab}\right)$ is full, the injective natural transformation

$$
0 \rightarrow-\otimes_{R} M \rightarrow-\otimes_{R} N
$$

comes from an injective module homomorphism $M \rightarrow N$. Since $-\otimes_{R} M \rightarrow$ $-\otimes_{R} N$ is injective, the map $M \rightarrow N$ is a pure embedding. Since $M$ is pure injective, it splits, so $M$ is isomorphic to a direct summand of $N$. So the natural transformation $-\otimes_{R} M \rightarrow-\otimes_{R} N$ splits, and $-\otimes_{R} M$ is a direct summand of an injective functor, and is hence injective. 


\subsection{Endomorphisms of injective objects}

Definition 3.3.1. A subobject $M$ of an object $I$ in $\mathcal{C}$ is essential if it has nonzero intersection with every nonzero subobject of $I$.

Lemma 3.3.2. Let $I$ be an object in a Grothendieck category $\mathcal{C}$.

(i) The intersection of a finite number of essential subobjects of $I$ is essential.

(ii) The inverse image of an essential subobject under an endomorphism of $I$ is essential.

(iii) The set of all elements of $\operatorname{End}(I)$ which annihilate some essential subobject of I is a two-sided ideal.

Proof. (i) If $M$ and $M^{\prime}$ are essential in $I$ then for any subobject $N$ of $I$,

$$
\left(M \cap M^{\prime}\right) \cap N=M \cap\left(M^{\prime} \cap N\right) \neq 0 .
$$

(ii) Let $f$ be an endomorphism of $I$. If $M$ is essential and $N$ is a subobject of $M$ satisfying $f^{-1}(M) \cap N=0$ then $M \cap f(N)=0$ and so $f(N)=0$. Hence

$$
N \subseteq f^{-1}(0) \cap N \subseteq f^{-1}(M) \cap N=0 .
$$

(iii) If $f$ and $f^{\prime}$ annihilate $M$ and $M^{\prime}$ then $f+f^{\prime}$ annihilates $M \cap M^{\prime}$. If $f$ is arbitrary and $f^{\prime}$ annihilates $M^{\prime}$ then $f \circ f^{\prime}$ annihilates $M^{\prime}$ and $f^{\prime} \circ f$ annihilates $f^{-1}\left(M^{\prime}\right)$.

Lemma 3.3.3. If $M$ is a subobject of $I$ and $M^{\prime}$ is maximal among subobjects of I satisfying $M \cap M^{\prime}=0$ then $M+M^{\prime}$ is an essential subobject of $I$.

P r o of. We begin by remarking that by the exactness of filtered colimits (see the remarks at the end of Section 3.1) and Zorn's lemma, every subobject $M^{\prime}$ satisfying $M \cap M^{\prime}=0$ is contained in a maximal such subobject, so that this lemma has content.

If $N$ is a subobject of $I$ satisfying $\left(M+M^{\prime}\right) \cap N=0$ then since $M \cap M^{\prime}$ is also zero, $M+M^{\prime}+N$ is an internal direct sum in $I$, and so also $M \cap$ $\left(M^{\prime}+N\right)=0$. By maximality we have $M^{\prime}+N=M^{\prime}$, and so $N$ is contained in $M^{\prime}$. So $N$ is contained in $\left(M+M^{\prime}\right) \cap N=0$.

We recall that a von Neumann regular ring is one in which given any element $x$ there exists $y$ satisfying $x y x=x$.

LEMma 3.3.4. In a von Neumann ring, every principal (left) ideal is generated by an idempotent, and is therefore a direct summand of $R$ (considered as a left $R$-module). Moreover, every finitely generated left ideal is principal.

The Jacobson radical of a von Neumann regular ring is zero.

Proof. If $x y x=x$, then the left ideal generated by $x$ is the same as the left ideal generated by $y x$, which is idempotent. 
To see that every finitely generated left ideal is principal, by induction it suffices to see that every two-generated ideal $R e+R a$ (with $e$ idempotent) is principal. Since $R e+R x=R e+R x(1-e)$, we may suppose that $x=x(1-e)$. Let $y$ be an element of $R$ such that $x y x=x$. Then $f=(1-e) y x$ is idempotent and $e f=f e=0$. Since $x=x f$ we have $R x=R f$, and so $R e+R x=R e+R f=R(e+f)$. This last equality follows from the fact that $e=e(e+f)$ and $f=f(e+f)$.

In general, the Jacobson radical of a ring is equal to the largest two-sided ideal consisting of elements $x$ such that $1-x$ is invertible. If $x=x y x$ is such an element in a von Neumann regular ring, then

$$
x=(1-x y)^{-1}(1-x y) x=(1-x y)^{-1}(x-x y x)=0 .
$$

It follows that the Jacobson radical of a von Neumann regular ring is zero.

The following theorem is essentially due to Lambek [24].

TheOREM 3.3.5. Let I be an injective object in a Grothendieck category. Let $J$ be the Jacobson radical of $\operatorname{End}(I)$. Then

(i) $\operatorname{End}(I) / J$ is a von Neumann regular ring.

(ii) $J$ consists of precisely those endomorphisms which annihilate some essential subobject of $I$.

(iii) Idempotents in the quotient ring $\operatorname{End}(I) / J$ lift to $\operatorname{End}(I)$.

(iv) Orthogonal idempotent decompositions of the identity element in the quotient ring $\operatorname{End}(I) / J$ lift to $\operatorname{End}(I)$.

In particular, if $I$ is indecomposable then $\operatorname{End}(I)$ is a local ring.

Proof. Let $J^{\prime}$ be the ideal named in (ii). We begin by proving that (i) holds with $J^{\prime}$ instead of $J$.

Let $x \in \operatorname{End}(I)$, and choose a subobject $M$ of $I$ maximal among subobjects satisfying $\operatorname{Ker}(x) \cap M=0$. Then by Lemma 3.3.3, $\operatorname{Ker}(x)+M$ is essential in $I$. Now $x$ restricts to an isomorphism between $M$ and $x(M) \subseteq I$. Its inverse extends to a map $y: I \rightarrow I$, since $I$ is injective.

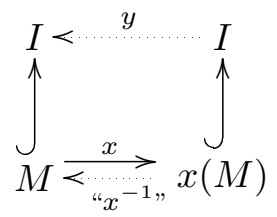

Now $x y x-x$ annihilates $\operatorname{Ker}(x)+M$, which is essential, so $x y x \equiv x \bmod$ $J^{\prime}$. Thus $\operatorname{End}(I) / J^{\prime}$ is a von Neumann regular ring. According to the above lemma, the Jacobson radical of a von Neumann regular ring is zero. It follows that $J \subseteq J^{\prime}$. 
Conversely, if $x \in J^{\prime}$ then $\operatorname{Ker}(x)$ is essential in $I$. Since

$$
\operatorname{Ker}(x) \cap \operatorname{Ker}(1-x)=0,
$$

this implies that $1-x$ is a monomorphism. Since $I$ is injective, the image of $1-x$ is a direct summand of $I$. It contains $\operatorname{Ker}(x)$, and is hence essential in $I$, so it must be equal to $I$. So $1-x$ is an automorphism of $I$ for every $x$ in $J^{\prime}$. If $\mathfrak{m}$ is a maximal (left) ideal in $\operatorname{End}(I)$ then $J^{\prime} \subseteq \mathfrak{m}$, since otherwise $1 \in J^{\prime}+\mathfrak{m}$, and then $1-x \in \mathfrak{m}$ for some $x \in J^{\prime}$. So we have $J=J^{\prime}$.

Next, we prove (iii). Suppose we are given $x \in \operatorname{End}(I)$ such that $x^{2}-x \in$ $J=J^{\prime}$. Then by definition of $J^{\prime}, K=\operatorname{Ker}\left(x^{2}-x\right)$ is essential in $I$. Let $I_{0}$ be the injective envelope of $x K$. Then $I$ decomposes as $I_{0} \oplus I_{1}$, and so $I_{0}=f I$ for a suitable idempotent $f \in \operatorname{End}(I)$. Now $f$ is not a lift of $x$, but we do have $x K=x^{2} K \subseteq f I$, so $f$ acts as the identity on $x K$. This implies that $(f x-x) K=0$, so that $f x-x \in J^{\prime}$. Set $e=f+f x(1-f)$. Then $e^{2}=e$, $e f=f$ and $f e=e$. Set

$$
K^{\prime}=x K+(1-f) I=x K \oplus I_{1} \subseteq I_{0} \oplus I_{1}=I .
$$

Then $K^{\prime}$ is essential in $I$, and

$$
\begin{aligned}
(e-f x) K^{\prime} & =(f+f x(1-f)-f x) K^{\prime}=(f-f x f) K^{\prime} \\
& =(f x-f x f x) K+(f-f x f)(1-f) I=\left(x-x^{2}\right) K+0=0 .
\end{aligned}
$$

So $e \equiv f x \equiv x \bmod J^{\prime}$, and $e$ is the required idempotent lift of $x$.

Finally, we prove (iv). Let $1=e_{1}+\ldots+e_{n}$ be an orthogonal idempotent decomposition of the identity element of $\operatorname{End}(I) / J$. In other words, the $e_{i}$ are idempotent, and for $i \neq j$ between 1 and $n, e_{i} e_{j}=e_{j} e_{i}=0$. Define idempotents $f_{i}^{\prime} \in \operatorname{End}(I)$ (these are not quite the idempotents we are after) inductively as follows. We set $f_{1}^{\prime}=1$, and for $i>1, f_{i}^{\prime}$ is any lift of $e_{i}+\ldots+e_{n}$ to an idempotent in the ring $f_{i-1}^{\prime} \operatorname{End}(I) f_{i-1}^{\prime}$. (Note that $f_{i-1}^{\prime} \operatorname{End}(I) f_{i-1}^{\prime}=$ $\operatorname{End}\left(f_{i-1}^{\prime} I\right)$ and $f_{i-1}^{\prime} I$ is injective; moreover,

$$
\begin{aligned}
e_{i}+\ldots+e_{n} \in\left(\operatorname{End}\left(f_{i-1}^{\prime} I\right)+J\right) / J \\
\left.\quad=\left(e_{i-1}+\ldots+e_{n}\right)(\operatorname{End}(I) / J)\left(e_{i-1}+\ldots+e_{n}\right) .\right)
\end{aligned}
$$

Then $f_{i}^{\prime} f_{i+1}^{\prime}=f_{i+1}^{\prime}=f_{i+1}^{\prime} f_{i}^{\prime}$. Define $f_{i}=f_{i}^{\prime}-f_{i+1}^{\prime}$ for $i<n$ and $f_{n}=f_{n}^{\prime}$. Then $f_{i}$ is an idempotent lift of $e_{i}$. Furthermore, if $1 \leq i<j \leq n$ then $f_{j}=f_{i+1}^{\prime} f_{j} f_{i+1}^{\prime}$, and so $f_{i} f_{j}=\left(f_{i}^{\prime}-f_{i+1}^{\prime}\right) f_{i+1}^{\prime} f_{j} f_{i+1}^{\prime}=0$. Similarly $f_{j} f_{i}$ $=0$.

COROLLARY 3.3.6. The endomorphism ring of a pure injective module satisfies the conclusion of the above theorem.

Proof. This follows from the above theorem, together with Theorem 3.2.1. 


\section{PHANTOM MAPS}

4.1. Topology. In topology, there are (at least) four separate notions of phantom map, namely two unstable ones and two stable ones. The first notion is that a map $f: X \rightarrow Y$ between CW complexes is phantom if its restriction to each skeleton $X^{n}$ is null homotopic. A good summary of what is known about phantom maps in this context can be found in the excellent survey article of McGibbon [25]. One feature of this definition is that the composite of any two phantom maps is null homotopic. If we want to extend this definition to maps between arbitrary topological spaces, it is easy to do so. We simply replace the finite-dimensional subcomplexes by maps from finite-dimensional complexes, and compose. If we do this, then the composite of two phantom maps is only weakly null homotopic. (We say that a map is weakly null homotopic if its composite with maps out of CW complexes are null homotopic.)

The second notion is also for CW complexes. A map $f: X \rightarrow Y$ is phantom in the second sense if its restriction to any finite subcomplex of $X$ is null homotopic. This agrees with the first notion if $X$ is of finite type (i.e., has only a finite number of cells of each dimension), but is in general weaker. There are more phantom maps in the second sense than in the first, and it is not known whether in this context there can be two phantom maps whose composite is not null homotopic. Again, we can extend to arbitrary topological spaces if we wish, by replacing finite subcomplexes by maps from finite complexes, and compose as before.

The third and fourth notions are the corresponding stable ones, which take place in a suitable category of spectra. A map $f: X \rightarrow Y$ between spectra is phantom in the third sense if its composite with any map from a finite spectrum to $X$ is null homotopic. This is equivalent to the statement that the corresponding map on homology theories $h_{f}: h_{X} \rightarrow h_{Y}$ is zero. Phantom maps in this context are studied extensively in Christensen and Strickland [15] and Neeman [26]. In fact, in [15] the discussion is axiomatized to provide a categorical framework for proving properties of phantom maps. Their notion of a Brown category is a particular type of triangulated category in which an appropriate version of the Brown representability axiom holds. In a Brown category, the composite of two phantom maps is always trivial. It turns out that not every triangulated category where one would like to discuss phantom maps is an example of a Brown category. It was observed by Neeman [26] that the derived category of unbounded complexes of modules over a polynomial ring in two variables over a field $k$ satisfies Brown representability if and only if $k$ is countable. In order to do this, he applied the theory of pure projective resolutions and quoted a theorem of Kaplansky on the 
projective dimensions of certain flat modules over such a ring (cf. Proposition 2.7.1).

Since there is a concept of finite-dimensional spectrum, there is a fourth notion of phantom map, which again takes place in the category of spectra, and which bears the same relation to the third definition as the first does to the second. Namely, a map $f: X \rightarrow Y$ of spectra is phantom if its composite with any map from a finite-dimensional spectrum to $X$ is null homotopic.

Some of the highpoints of the topological theory are the following. We describe these for the first definition given above, but there are similar statements for the others. For every CW complex $X$, there is a phantom map $X \rightarrow Z$ which is weakly universal, in the sense that every phantom map out of $X$ factors through $Z$, but not uniquely. The complex $Z$ can be taken to be $\bigvee_{n} \Sigma X^{n}$, the wedge of the suspensions of the skeleta of $X$. In particular, since $Z$ is a wedge of finite-dimensional complexes, there are no nontrivial phantom maps out of $Z$. This shows that the composite of two phantom maps is null homotopic.

A map $X \rightarrow Y$ between connected nilpotent spaces of finite type is phantom if and only if the composite $X \rightarrow Y \rightarrow \widehat{Y}$ is null homotopic (Zabrodsky [33]). Here, $\widehat{Y}$ denotes the Sullivan profinite completion of $Y$. It is worth discussing at this stage what to expect to be the analog in representation theory of the Sullivan profinite completion. Given a vector space $V$ over a field $k$, one might think of taking all the finite-dimensional quotients of $V$ and then taking the inverse limit of this system. Since the finite-dimensional quotients of $V$ are exactly the duals of the finite-dimensional subspaces of $V^{*}$, this inverse system is dual to the direct system of finite-dimensional subspaces of $V^{*}$. It follows that the inverse limit is precisely $V^{* *}$, and so the inclusion of $V$ into $V^{* *}$ should be regarded as a profinite completion. Proposition 4.2.2 below is then the analog of the above statement about the Sullivan profinite completion.

A classical example of Brayton Gray [17] of a phantom map in topology (in the first of the above senses) is as follows. He proved that there are an uncountable number of homotopy classes of phantom maps from $\mathbb{C} P^{\infty}$ to $S^{3}$. In contrast, in representation theory there can never be a phantom map whose target is a finite-dimensional module, by Theorem 4.2.5.

4.2. Representation theory. Let $k$ be a field of characteristic $p$ and let $G$ be a finite group. We say that a map of $k G$-modules $M \rightarrow N$ is phantom if its restriction to every finitely generated submodule of $M$ factors through a projective $k G$-module. Note that in this context, finitely generated, finitely presented and finite-dimensional are all the same condition. The results showing the relationship between phantom maps and purity are similar to results obtained by Christensen in [14]. 
We write $\operatorname{PHom}_{k G}(M, N)$ for the linear subspace of $\operatorname{Hom}_{k G}(M, N)$ consisting of maps which factor through some projective module, and $\underline{\operatorname{Hom}}_{k G}(M, N)$ for the quotient

$$
\underline{\operatorname{Hom}}_{k G}(M, N)=\operatorname{Hom}_{k G}(M, N) / \operatorname{PHom}_{k G}(M, N) .
$$

We write $\operatorname{Ph}_{k G}(M, N)$ for the space of phantom maps from $M$ to $N$ and $\underline{\mathrm{Ph}}_{k G}(M, N)$ for the quotient

$$
\underline{\mathrm{Ph}}_{k G}(M, N)=\mathrm{Ph}_{k G}(M, N) / \mathrm{PHom}_{k G}(M, N) .
$$

We recall that the stable module category $\operatorname{StMod}(k G)$ has as its objects the (not necessarily finitely generated) $k G$-modules, and as its arrows the spaces $\underline{\operatorname{Hom}}_{k G}(M, N)$. We write $\operatorname{stmod}(k G)$ for the corresponding category in which the objects are the finitely generated $k G$-modules, with the same definition for the maps.

The categories $\operatorname{StMod}(k G)$ and $\operatorname{stmod}(k G)$ are not abelian categories, but rather they have a natural structure of triangulated category in which the translation functor is $\Omega^{-1}$ and the distinguished triangles

$$
M_{1} \rightarrow M_{2} \rightarrow M_{3} \rightarrow \Omega^{-1} M_{1}
$$

come from short exact sequences

$$
0 \rightarrow M_{1} \rightarrow M_{2} \rightarrow M_{3} \rightarrow 0
$$

in the corresponding module category.

Any map $M \rightarrow N$ of $k G$-modules can be completed to a distinguished triangle in either direction. Completing to a triangle

$$
\Omega N \rightarrow X \rightarrow M \rightarrow N
$$

displays the natural isomorphism

$$
\underline{\operatorname{Hom}}_{k G}(M, N) \cong \operatorname{Ext}_{k G}^{1}(M, \Omega N) .
$$

Lemma 4.2.1. Let $M$ be a $k G$-module. Then

(i) $\Omega\left(M^{*}\right)$ and $\left(\Omega^{-1}(M)\right)^{*}$ are isomorphic in $\operatorname{StMod}(k G)$.

(ii) A $k G$-module $M$ is pure projective if and only if $\Omega(M)$ is pure projective.

(iii) A $k G$-module $M$ is pure injective if and only if $\Omega(M)$ is pure injective.

(iv) A sequence of $k G$-modules

$$
0 \rightarrow M_{1} \rightarrow M_{2} \rightarrow M_{3} \rightarrow 0
$$

is pure exact if and only if the translated sequence

$$
0 \rightarrow \Omega M_{1} \rightarrow \Omega M_{2} \oplus \text { (projective) } \rightarrow \Omega M_{3} \rightarrow 0
$$

is pure exact. 
Proof. (i) This follows from the fact that the dual of a monomorphism $M \rightarrow I$ of $M$ into an injective module gives an epimorphism from the projective module $I^{*}$ onto $M^{*}$.

(ii), (iii) and (iv) are easy to prove and left to the reader.

Proposition 4.2.2. Let $\zeta: M \rightarrow N$ represent an element

$$
\bar{\zeta} \in \underline{\operatorname{Hom}}_{k G}(M, N) \cong \operatorname{Ext}_{k G}^{1}(M, \Omega N) .
$$

Then the following are equivalent:

(i) $\bar{\zeta}$ lies in $\underline{\mathrm{Ph}}_{k G}(M, N)$.

(ii) The corresponding extension $0 \rightarrow \Omega N \rightarrow X \rightarrow M \rightarrow 0$ is pure exact.

(iii) The composite of $\zeta$ with the natural inclusion of $N$ into $N^{* *}$ factors through a projective module.

Pro of. The equivalence of (i) and (ii) follows from the long exact Hom sequence of a triangle in $\operatorname{StMod}(k G)$. Namely, the given extension corresponds to a triangle

$$
\Omega N \rightarrow X \rightarrow M \rightarrow N .
$$

So the given map from $M$ to $N$ is phantom if and only if for every map from a finitely generated module $Y$ to $M$, the composite map from $Y$ to $N$ factors through a projective module, which is the same as saying that it is zero in $\operatorname{StMod}(k G)$. By the long exact sequence, this happens if and only if it lifts in $\operatorname{StMod}(k G)$ to a map from $Y$ to $X$. Since maps which factor through a projective module always lift, this implies that the map lifts in $\operatorname{Mod}(k G)$.

By Corollary 2.3.4, $N^{* *}$ is pure injective, and so by Lemma 4.2.1, $\Omega\left(N^{* *}\right)$ is also pure injective. If (ii) holds, then we have a diagram

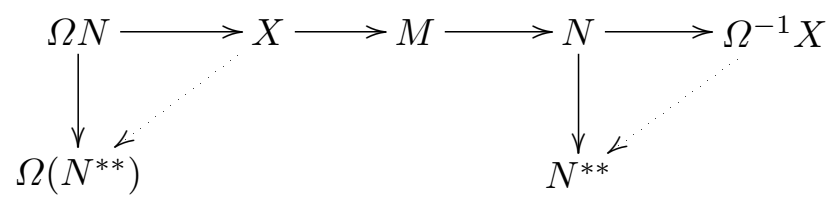

The map $\Omega N \rightarrow \Omega\left(N^{* *}\right)$ lifts to a map $X \rightarrow \Omega\left(N^{* *}\right)$, and so the map $N \rightarrow N^{* *}$ lifts to $\Omega^{-1} X \rightarrow N^{* *}$. Thus the composite $M \rightarrow N \rightarrow N^{* *}$ factors through a projective module, and so (iii) holds.

Finally, we prove that (iii) implies (ii). By Theorem 2.4.2, the inclusion of $N$ into $N^{* *}$ is a pure monomorphism. So the map $\Omega N \rightarrow \Omega(N)^{* *} \cong \Omega\left(N^{* *}\right)$ is a pure monomorphism. Since the composite $\Omega M \rightarrow \Omega N \rightarrow \Omega\left(N^{* *}\right)$ factors through a projective module, we obtain a factorization $\Omega N \rightarrow X \rightarrow$ $\Omega\left(N^{* *}\right)$ of the pure monomorphism $\Omega N \rightarrow \Omega\left(N^{* *}\right)$. So the map $\Omega N \rightarrow X$ is a pure monomorphism. 
COROLLARY 4.2.3. There are natural isomorphisms

$$
\underline{\operatorname{Ph}}_{k G}(M, N) \cong \operatorname{Pext}_{k G}^{1}(M, \Omega N) \cong \operatorname{Pext}_{k G}^{1}\left(\Omega^{-1} M, N\right) \text {. }
$$

Proof. This follows from the equivalence between (i) and (ii) in the above proposition.

It is also easy to read off from Proposition 4.2.2 the description of (weakly) universal phantom maps out of a module given in [16]. Namely, if we express a $k G$-module $M$ as a filtered colimit of finite-dimensional modules $M=\lim _{\longrightarrow} M_{\alpha}$, then rotating the distinguished triangle coming from the short exact sequence

$$
0 \rightarrow K \rightarrow \bigoplus_{\alpha} M_{\alpha} \rightarrow M \rightarrow 0
$$

we obtain a map $M \rightarrow \Omega^{-1} K$. By Lemma 2.2 .2 and Proposition 4.2.2, this is a phantom map. Moreover, given any phantom map $f: M \rightarrow N$, the composite of $f$ with the map from the direct sum to $M$ factors through a projective module, and so $f$ factors (but not by any means uniquely) through $M \rightarrow \Omega^{-1} K$.

In a similar way, we obtain (weakly) universal phantom maps into a $k G$-module $N$. Namely, we apply Theorem 2.4 .2 to see that we have a pure exact sequence $0 \rightarrow N \rightarrow N^{* *} \rightarrow N^{* *} / N \rightarrow 0$. Rotating the corresponding distinguished triangle, we have a phantom map $\Omega\left(N^{* *} / N\right) \rightarrow N$. By Proposition 4.2.2, given any phantom map $f: M \rightarrow N$, the composite with the inclusion of $N$ into $N^{* *}$ factors through a projective module, and so $f$ factors (but not uniquely) through $\Omega\left(N^{* *} / N\right) \rightarrow N$.

TheOREM 4.2.4. For a $k G$-module $M$, the following are equivalent:

(i) $\underline{\mathrm{Ph}}_{k G}(M, N)=0$ for all $k G$-modules $N$.

(ii) $M$ is pure projective.

(iii) $M$ is isomorphic to a direct sum of finitely generated modules.

Proof. The equivalence of (i) and (ii) follows from the isomorphism between the first and second terms of Corollary 4.2.3, while the equivalence of (ii) and (iii) is given in Theorem 2.2.4.

TheOREM 4.2.5. For a $k G$-module $N$, the following are equivalent:

(i) $\underline{\mathrm{Ph}}_{k G}(M, N)=0$ for all $k G$-modules $M$.

(ii) $N$ is pure injective.

(iii) $N$ is isomorphic to a direct summand of a direct product of finitely generated modules.

(iv) $N$ is isomorphic to a direct summand of the dual of some module.

(v) The natural monomorphism $N \rightarrow N^{* *}$ splits. 
If $N$ satisfies these equivalent conditions, then the endomorphism ring $\operatorname{End}_{k G}(N)$ has the following properties:

(i) The quotient of $\operatorname{End}_{k G}(N)$ by its Jacobson radical $J$ is a von Neumann regular ring.

(ii) Orthogonal idempotent decompositions of the identity element in the quotient ring $\operatorname{End}_{k G}(N) / J$ lift to orthogonal idempotent decompositions of the identity element in $\operatorname{End}_{k G}(N)$.

In particular, if $N$ is indecomposable then $\operatorname{End}_{k G}(N)$ is a (not necessarily commutative) local ring, and so is $\underline{\operatorname{End}}_{k G}(N)$.

Proof. The equivalence of (i) and (ii) follows from the isomorphism between the first and third terms in Corollary 4.2.3. The equivalence of (ii) and (iii) follows from Corollary 2.4.5. The equivalence of (ii) and (iv) follows from Corollary 2.4.4. Finally, the equivalence of (ii) and (v) follows from Corollary 2.4.3.

The last part of this theorem follows by applying Corollary 3.3.6.

4.3. Operations on $k G$-modules. In this section we examine the effect on purity of the usual operations on modules for group algebras.

Proposition 4.3.1. If $H$ is a subgroup of a finite group $G$ then induction and restriction between $k H$-modules and $k G$-modules

(i) take pure projective modules to pure projective modules,

(ii) take pure injective modules to pure injective modules, and

(iii) take pure exact sequences to pure exact sequences.

Proof. Induction and restriction take finitely presented (i.e., finite-dimensional) modules to finitely presented modules. So (i) follows from Theorem 2.2.3, and (ii) from Corollary 2.4.5. (iii) can be obtained using the Nakayama relations (Frobenius reciprocity). Namely, if $M$ is a $k G$-module and $N$ is a $k H$-module then

$$
\begin{aligned}
& \operatorname{Hom}_{k G}\left(M, N \uparrow^{G}\right) \cong \operatorname{Hom}_{k H}\left(M \downarrow_{H}, N\right), \\
& \operatorname{Hom}_{k G}\left(N \uparrow^{G}, M\right) \cong \operatorname{Hom}_{k H}\left(N, M \downarrow_{H}\right) .
\end{aligned}
$$

Proposition 4.3.2. Let $H$ be a subgroup of a finite group $G$.

(i) If $M$ and $N$ are pure projective $k G$-modules then the tensor product $M \otimes_{k} N$ is a pure projective $k G$-module.

(ii) If $M$ is a pure projective $k H$-module then the tensor induced module $M{ }_{\Phi}{ }^{G}$ is pure projective.

Proof. Both parts follow from Proposition 2.2.3 and an analysis of tensor products of direct sums. 
Next, we investigate the varieties of modules in a pure exact sequence, in the sense of Benson, Carlson and Rickard [10].

LEMmA 4.3.3. Let $K$ be an extension field of $k$ and $G$ be a finite group. If $0 \rightarrow A \rightarrow B \rightarrow C \rightarrow 0$ is a pure exact sequence of $k G$-modules, then

$$
0 \rightarrow K \otimes_{k} A \rightarrow K \otimes_{k} B \rightarrow K \otimes_{k} C \rightarrow 0
$$

is a pure exact sequence of $K G$-modules.

Proof. If $M$ is any right $K G$-module then we have a natural isomorphism $M \otimes_{K G}\left(K \otimes_{k}-\right) \cong M \otimes_{k G}-$, so the lemma follows directly from the definition of pure exactness.

THEOREM 4.3.4. Let $k$ be an algebraically closed field of characteristic $p$ and $G$ be a finite group. If $0 \rightarrow A \rightarrow B \rightarrow C \rightarrow 0$ is a pure exact sequence of $k G$-modules then

$$
\mathcal{V}_{G}(B)=\mathcal{V}_{G}(A) \cup \mathcal{V}_{G}(C) .
$$

Proof. It follows from Theorems 10.5 and 10.6 of [10] together with Proposition 4.3.1 above that it suffices to prove this statement for rank varieties in the case where $G$ is an elementary abelian $p$-group. In this case, to evaluate the rank variety, the recipe is to tensor with an algebraically closed extension field $K$ of $k$ of sufficiently large transcendence degree, and then restrict to a cyclic shifted subgroup of the group algebra $K E$. By Lemma 4.3.3, tensoring with $K$ takes pure exact sequences to pure exact sequences. The argument used to prove Proposition 4.3.1 shows that restriction to a cyclic shifted subgroup also takes pure exact sequences to pure exact sequences. All modules for a cyclic shifted subgroup are pure projective (the group algebra has finite representation type), so pure exact sequences for such a subgroup split. So the middle term fails to be free if and only if at least one of the outside terms does. This completes the proof of the theorem.

4.4. Modules with flat cohomology. In this section we examine the theory of pure projective resolutions for $k G$-modules $M$ such that $\widehat{H}^{*}(G, M)$ is flat as an $\widehat{H}^{*}(G, k)$-module. Our main tool is a spectral sequence argument. The spectral sequence in question has convergence problems unless $\widehat{H}^{*}(G, M)$ also has finite projective dimension as an $\widehat{H}^{*}(G, k)$-module, so we make this part of the hypothesis.

THEOREM 4.4.1. For a short exact sequence of $k G$-modules

$$
0 \rightarrow M_{1} \rightarrow M_{2} \rightarrow M_{3} \rightarrow 0
$$

the following are equivalent.

(i) The sequence is pure exact. 
(ii) For all finitely generated $k G$-modules $N$,

$$
0 \rightarrow \operatorname{Hom}_{k G}\left(N, M_{1}\right) \rightarrow \operatorname{Hom}_{k G}\left(N, M_{2}\right) \rightarrow \operatorname{Hom}_{k G}\left(N, M_{3}\right) \rightarrow 0
$$

is exact.

(iii) For all finitely generated $k G$-modules $N$,

$$
0 \rightarrow \operatorname{Ext}_{k G}^{*}\left(N, M_{1}\right) \rightarrow \operatorname{Ext}_{k G}^{*}\left(N, M_{2}\right) \rightarrow \operatorname{Ext}_{k G}^{*}\left(N, M_{3}\right) \rightarrow 0
$$

is a pure exact sequence of graded $H^{*}(G, k)$-modules.

(iv) For all finitely generated $k G$-modules $N$,

$$
0 \rightarrow \widehat{\operatorname{Ext}}_{k G}^{*}\left(N, M_{1}\right) \rightarrow \widehat{\operatorname{Ext}}_{k G}^{*}\left(N, M_{2}\right) \rightarrow \widehat{\operatorname{Ext}}_{k G}^{*}\left(N, M_{3}\right) \rightarrow 0
$$

is a pure exact sequence of graded $\widehat{H}^{*}(G, k)$-modules.

Proof. Since (iii) $\Rightarrow$ (ii) $\Rightarrow$ (i) is obvious, we prove that (i) $\Rightarrow$ (iv) $\Rightarrow$ (iii). We begin with (iv) $\Rightarrow$ (iii). In positive degrees, $\operatorname{Ext}_{k G}^{*}\left(N, M_{i}\right)(i \in\{1,2,3\})$ and $\widehat{\operatorname{Ext}}_{k G}^{*}\left(N, M_{i}\right)$ agree, so this amounts to examining what is happening in degree zero. Now the natural map from $\operatorname{Hom}_{k G}\left(N, M_{i}\right)=\operatorname{Ext}_{k G}^{0}\left(N, M_{i}\right)$ to $\widehat{\operatorname{Ext}}_{k G}^{0}\left(N, M_{i}\right)$ is surjective, and the kernel is $\operatorname{PHom}_{k G}\left(N, M_{i}\right)$, the projective homomorphisms (see Section 4.2). Not only is $\operatorname{PHom}_{k G}(N,-)$ exact, but it consists of elements whose product with any positive degree element of $H^{*}(G, k)$ is zero. So as an $H^{*}(G, k)$-module, $\operatorname{Ext}_{k G}^{*}\left(N, M_{i}\right)$ decomposes as a direct sum of $\operatorname{PHom}_{k G}\left(N, M_{i}\right)$ and the nonnegative degree part of $\widehat{\operatorname{Ext}}_{k G}^{*}\left(N, M_{i}\right)$. We may choose a decomposition which respects the maps in the sequence. To prove (i) $\Rightarrow$ (iv), we suppose that we are given a finitely presented graded $\widehat{H}^{*}(G, k)$-module $X$ with generators $x_{1}, \ldots, x_{r}$ in degrees $m_{1}, \ldots, m_{r}$ and relators $y_{1}, \ldots, y_{s}$ in degrees $n_{1}, \ldots, n_{s}$. We form modules

$$
A=\bigoplus_{i=1}^{s} \Omega^{n_{i}}(k), \quad B=\bigoplus_{j=1}^{r} \Omega^{m_{j}}(k) .
$$

Expressing each $y_{i}$ as a linear combination of the $x_{j}$ with coefficients in $\widehat{H}^{*}(G, k)$,

$$
y_{i}=\sum_{j} \gamma_{i j} x_{j}
$$

we have $\operatorname{deg}\left(\gamma_{i j}\right)=n_{i}-m_{j}$. So $\gamma_{i j}$ can be represented by a $k G$-module homomorphism $\widehat{\gamma}_{i j}: \Omega^{n_{i}}(k) \rightarrow \Omega^{m_{j}}(k)$. This way, we obtain a $k G$-module homomorphism $\gamma=\left(\widehat{\gamma}_{i j}\right): A \rightarrow B$. We complete $\gamma$ to a triangle in $\operatorname{stmod}(k G)$ :

$$
A \stackrel{\gamma}{\longrightarrow} B \stackrel{\theta}{\longrightarrow} C \rightarrow \Omega^{-1} A \text {. }
$$

Given a homomorphism of graded $\widehat{H}^{*}(G, k)$-modules

$$
\phi: X \rightarrow \widehat{\operatorname{Ext}}_{k G}^{*}\left(N, M_{3}\right) \cong \widehat{H}^{*}\left(G, \operatorname{Hom}_{k}\left(N, M_{3}\right)\right),
$$


we obtain corresponding $k G$-module homomorphisms

$$
\widehat{\phi\left(x_{i}\right)}: \Omega^{m_{i}}(k) \rightarrow \operatorname{Hom}_{k}\left(N, M_{3}\right)
$$

and hence a $k G$-module homomorphism $\widehat{\phi}: B \rightarrow \operatorname{Hom}_{k}\left(N, M_{3}\right)$ whose composite with $\gamma$ factors through a projective module. This gives us a $k G$-module homomorphism $\psi: C \rightarrow \operatorname{Hom}_{k}\left(N, M_{3}\right)$ or equivalently a homomorphism $C \otimes_{k} N \rightarrow M_{3}$.

Since $0 \rightarrow M_{1} \rightarrow M_{2} \rightarrow M_{3} \rightarrow 0$ is pure exact, this map lifts to a homomorphism $C \otimes_{k} N \rightarrow M_{2}$. This implies that $\psi$ lifts to a homomorphism $\psi^{\prime}: C \rightarrow \operatorname{Hom}_{k}\left(N, M_{2}\right)$. This in turn means that we have a map

$$
\psi^{\prime} \circ \theta=\widehat{\phi}^{\prime}: B \rightarrow \operatorname{Hom}_{k}\left(N, M_{2}\right)
$$

whose composite with $\gamma$ is zero in $\operatorname{stmod}(k G)$, and whose composite with $\operatorname{Hom}_{k}\left(N, M_{2}\right) \rightarrow \operatorname{Hom}_{k}\left(N, M_{3}\right)$ is equal to $\widehat{\phi}$. Finally, this gives us the required map of $\widehat{H}^{*}(G, k)$-modules

$$
\phi^{\prime}: X \rightarrow \widehat{\operatorname{Ext}}_{k G}^{*}\left(N, M_{2}\right)
$$

whose composite with $\widehat{\operatorname{Ext}}_{k G}^{*}\left(N, M_{2}\right) \rightarrow \widehat{\operatorname{Ext}}_{k G}^{*}\left(N, M_{3}\right)$ is equal to $\phi$.

TheOREM 4.4.2. Let $G$ be a finite p-group and let $k$ be a field of characteristic $p$. Let $M$ be a $k G$-module with the property that $\widehat{H}^{*}(G, M)$ is flat and of finite projective dimension as a graded $\widehat{H}^{*}(G, k)$-module. Then for any finitely generated $k G$-module $N$, the map

$$
\widehat{\operatorname{Ext}}_{k G}^{*}(N, k) \otimes_{\widehat{H}^{*}(G, k)} \widehat{H}^{*}(G, M) \rightarrow \widehat{\operatorname{Ext}}_{k G}^{*}(N, M)
$$

is an isomorphism.

Proof. Let

$$
0 \rightarrow F_{r} \rightarrow \ldots \rightarrow F_{1} \rightarrow F_{0} \rightarrow \widehat{H}^{*}(G, M) \rightarrow 0
$$

be a free resolution of finite length for $\widehat{H}^{*}(G, M)$ as a graded $\widehat{H}^{*}(G, k)$ module. We form an exact sequence

$$
R_{r} \rightarrow \ldots \rightarrow R_{1} \rightarrow R_{0} \rightarrow M \rightarrow 0
$$

where each $R_{j}$ is a direct sum of modules of the form $\Omega^{n} k(n \in \mathbb{Z})$ and projective modules, and such that applying $\widehat{H}^{*}(G,-)$ gives the above free resolution, $\widehat{H}^{*}\left(G, R_{j}\right) \cong F_{j}$. Then the kernel $K$ of the first map in this sequence has the property that $\widehat{H}^{*}(G, K)=0$. Since $G$ is a $p$-group, this implies that $K$ is projective as a $k G$-module. Projective $k G$-modules are injective, so $K$ splits off the first term above. So we may assume that the first map in the above sequence is injective.

Let

$$
\ldots \rightarrow P_{1} \rightarrow P_{0} \rightarrow P_{-1} \rightarrow \ldots
$$


be a complete (Tate) resolution of the finitely generated $k G$-module $N$. In other words, it is an exact sequence of projectives, infinite in both directions, such that the image of $P_{0} \rightarrow P_{-1}$ is isomorphic to $N$. We form the double complex

$$
E_{s t}^{0}=\operatorname{Hom}_{k G}\left(P_{-s}, R_{t}\right) .
$$

This double complex gives us two spectral sequences. Since there is a finite number of rows in the double complex, there are no convergence problems.

In the spectral sequence in which we do the differential coming from the complex of $R_{j}$ 's first, we have

$$
E_{s t}^{1} \cong \begin{cases}\operatorname{Hom}_{k G}\left(P_{-s}, M\right), & t=0, \\ 0, & t>0,\end{cases}
$$

and so

$$
E_{s t}^{2} \cong \begin{cases}\widehat{\operatorname{Ext}}_{k G}^{-s}(N, M), & t=0 \\ 0, & t>0 .\end{cases}
$$

On the other hand, if we do the differential coming from the Tate resolution first, then we have

$$
E_{s t}^{1} \cong \widehat{\operatorname{Ext}}_{k G}^{-s}\left(N, R_{t}\right), \quad E_{* t}^{1} \cong \widehat{\operatorname{Ext}}_{k G}^{*}(N, k) \otimes_{\widehat{H}^{*}(G, k)} F_{t} .
$$

This last isomorphism follows from the facts that $N$ is finitely generated and $F_{t}$ is free over $\widehat{H}^{*}(G, k)$ on basis elements corresponding to the summands of $R_{t}$. Finally, since $\widehat{H}^{*}(G, M)$ is flat as an $\widehat{H}^{*}(G, k)$-module, and this complex calculates Tor, we have

$$
E_{* t}^{2} \cong \begin{cases}\widehat{\operatorname{Ext}}_{k G}^{*}(N, k) \otimes_{\widehat{H}^{*}(G, k)} \widehat{H}^{*}(G, M), & t=0, \\ 0, & t>0 .\end{cases}
$$

The two spectral sequences must converge to the same answer, and an examination of edge homomorphisms shows that the isomorphism we get this way comes from the obvious map.

The following is a corollary of the proof, rather than the statement of the above theorem.

COROLlaRY 4.4.3. Under the assumptions of Theorem 4.4.2, the exact sequence

$$
0 \rightarrow R_{r} \rightarrow \ldots \rightarrow R_{1} \rightarrow R_{0} \rightarrow M \rightarrow 0
$$

described in the proof is a pure projective resolution of $M$.

Pr o of. For any finitely generated $k G$-module $N$, the sequence

$$
0 \rightarrow \widehat{\operatorname{Ext}}_{k G}^{*}\left(N, R_{r}\right) \rightarrow \ldots \rightarrow \widehat{\operatorname{Ext}}_{k G}^{*}\left(N, R_{0}\right) \rightarrow \widehat{\operatorname{Ext}}_{k G}^{*}(N, M) \rightarrow 0
$$

is the same as the sequence 


$$
\begin{aligned}
& 0 \rightarrow \widehat{\operatorname{Ext}}_{k G}^{*}(N, k) \otimes_{\widehat{H}^{*}(G, k)} \widehat{H}^{*}\left(G, R_{t}\right) \rightarrow \ldots \rightarrow \\
& \widehat{\operatorname{Ext}}_{k G}^{*}(N, k) \otimes_{\widehat{H}^{*}(G, k)} \widehat{H}^{*}\left(G, R_{0}\right) \rightarrow \widehat{\operatorname{Ext}}_{k G}^{*}(N, k) \otimes_{\widehat{H}^{*}(G, k)} \widehat{H}^{*}(G, M) \rightarrow 0 .
\end{aligned}
$$

Since $\widehat{H}^{*}(G, M)$ is a flat $\widehat{H}^{*}(G, k)$-module, this sequence is exact. The corollary now follows from the implication (iv) $\Rightarrow$ (i) of Theorem 4.4.1.

COROLlary 4.4.4. Let $G$ be a finite p-group and $k$ a field of characteristic $p$. Let $M$ be a $k G$-module such that $\widehat{H}^{*}(G, M)$ is flat and of nonzero finite projective dimension over $\widehat{H}^{*}(G, k)$. Then the pure projective dimension (ppd) of $M$ as a $k G$-module is equal to the projective dimension (pd) of $\widehat{H}^{*}(G, M)$ as an $\widehat{H}^{*}(G, k)$-module.

Pr o o f. We have $\operatorname{pd}_{\widehat{H}^{*}(G, k)}\left(\widehat{H}^{*}(G, M)\right) \leq \operatorname{ppd}_{k G}(M)$ by the implication (i) $\Rightarrow$ (iv) of Theorem 4.4.1, together with the fact that for a flat module the projective dimension is equal to the pure projective dimension (Proposition 2.7.1). We have $\operatorname{ppd}_{k G}(M) \leq \operatorname{fd}_{\widehat{H}^{*}(G, k)}\left(\widehat{H}^{*}(G, M)\right)$ by Corollary 4.4.3 (here, fd denotes the minimal length of a free resolution). Now $\operatorname{pd}_{\widehat{H}^{*}(G, k)}\left(\widehat{H}^{*}(G, M)\right)=\mathrm{fd}_{\widehat{H}^{*}(G, k)}\left(\widehat{H}^{*}(G, M)\right)$ by the Eilenberg swindle.

REMARK 4.4.5. The principal examples of modules with flat cohomology we have in mind are certain of Rickard's idempotent modules (see Section 5.4), but flat cohomology seems to be the correct generality for the proof.

4.5. Pure global dimension of $k G$. In this section we describe what is known about the pure global dimension of group algebras of finite groups.

LEMMA 4.5.1. Let $k$ be a field and $G$ be a finite group. Let $M$ be a $k G$ module which is projective relative to a subgroup $D$. Then the pure projective dimension of $M$ is the same as the pure projective dimension of $M \downarrow_{D}$.

Proof. This follows from the fact that induction and restriction between $G$ and $D$ take pure projective resolutions to pure projective resolutions and $M$ is a summand of $M \downarrow_{D} \uparrow^{G}$.

Proposition 4.5.2. Let $k$ be a field of characteristic $p$ and let $G$ be a finite group with $P$ a Sylow p-subgroup of $G$. Then the pure global dimension of $k G$ is the same as the pure global dimension of $k P$.

Pr o o f. Every $k G$-module is projective relative to $P$, so this follows from Lemma 4.5.1.

TheOREM 4.5.3. Let $k$ be a field of characteristic $p$ and cardinality $\aleph_{t}$.

(i) If $p=0$ or $G$ has (possibly trivial) cyclic Sylow p-subgroups then $k G$ has pure global dimension zero. 
(ii) If $p$ is odd and $G$ has noncyclic Sylow p-subgroups then the pure global dimension of $k G$ is $t+1$. Here and in the remaining parts of this theorem, if $t$ is infinite then $t+1$ is taken to mean $\infty$.

(iii) If $p=2$ and $G$ has Sylow 2-subgroups which are not cyclic, dihedral, semidihedral or generalized quaternion, then the pure global dimension of $k G$ is $t+1$.

(iv) If $p=2$ and $G$ has Sylow 2 -subgroups isomorphic to $\mathbb{Z} / 2 \times \mathbb{Z} / 2$ then the pure global dimension of $k G$ is equal to one if $k$ is countable, and two if $k$ is uncountable.

(v) In the remaining cases in characteristic two, namely when the Sylow 2 -subgroup is dihedral of order greater than four, semidihedral or generalized quaternion, then the pure global dimension of $k G$ is equal to one if $k$ is countable, two if $k$ has cardinality $\aleph_{1}$, and is unknown (but somewhere between 2 and $t+1$ ) if $k$ has cardinality $\aleph_{t}$ with $t>1$.

Pr o o f. By Proposition 4.5.2, we may replace $G$ by its Sylow $p$-subgroup $P$ and assume that $G=P$ is a $p$-group. In this case, $k P$ is a finitedimensional local algebra over $k$. If $P$ is a (possibly trivial) cyclic group then by a theorem of Auslander [2], every module is a direct sum of finitedimensional modules and $k P$ has pure global dimension zero. This takes care of case (i). In cases (ii) and (iii), $k P$ has wild representation type, and the statement follows from Theorem 4.1 of Baer and Lenzing [4]. In fact in this case $k P$ has as a quotient either the four-dimensional algebra

$$
k[X, Y, Z] /(X, Y, Z)^{2}
$$

or the five-dimensional algebra

$$
k[X, Y] /\left(X^{2}, X Y^{2}, Y^{3}\right) .
$$

These are the algebras $R_{1}$ and the case $\lambda=1$ of $R_{4}$ described in the proof of that theorem. In case (iv), it follows from Corollary 2.4 of [4] that the pure global dimension of $k P$ is the same as that of $k P / \operatorname{Soc}(k P)$. This is a radical square zero algebra of tame representation type, so by Theorem 3.4 of Baer, Brune and Lenzing [3], it has pure global dimension either one or two depending on whether $k$ is countable or uncountable (i.e., $t=0$ or $t \geq 1$ ). Finally, in case (v), $P$ has $\mathbb{Z} / 2 \times \mathbb{Z} / 2$ as a quotient group, so that by Corollary 2.3(i) of [4] the pure global dimension is at least that of case (iv).

4.6. Composites of phantom maps. We write $\underline{\mathrm{Ph}}_{k G}^{n}(M, N)$ for the subspace of $\underline{\operatorname{Hom}}_{k G}(M, N)$ consisting of maps which may be written as a composite of $n$ phantom maps.

THEOREM 4.6.1. There is a natural isomorphism between $\underline{\mathrm{Ph}}_{k G}^{n}(M, N)$ and the image of the natural map 


$$
\operatorname{Pext}_{k G}^{n}\left(M, \Omega^{n} N\right) \rightarrow \operatorname{Ext}_{k G}^{n}\left(M, \Omega^{n} N\right) .
$$

In case $n=1$, this map is injective, and gives the isomorphism

$$
\underline{\mathrm{Ph}}_{k G}(M, N) \cong \operatorname{Pext}_{k G}^{1}(M, \Omega N)
$$

of Corollary 4.2.3.

Pr o o f. Let

$$
M=M_{0} \stackrel{f_{0}}{\longrightarrow} M_{1} \stackrel{f_{1}}{\longrightarrow} \ldots \stackrel{f_{n-1}}{\longrightarrow} M_{n}=N
$$

be phantom maps with composite $f: M \rightarrow N$. Complete the phantom map $f_{i}: M_{i} \rightarrow M_{i+1}$ to a triangle

$$
\Omega M_{i+1} \rightarrow X_{i} \rightarrow M_{i} \rightarrow M_{i+1} .
$$

Translating, and using Proposition 4.2.2, we see that there is a pure exact sequence

$$
0 \rightarrow \Omega^{i+1} M_{i+1} \rightarrow \Omega^{i} X_{i} \oplus \text { (projective) } \rightarrow \Omega^{i} M_{i} \rightarrow 0
$$

representing the image of $f_{i}$ under the isomorphism

$$
\underline{\operatorname{Hom}}_{k G}\left(M_{i}, M_{i+1}\right) \cong \operatorname{Ext}_{k G}^{1}\left(\Omega^{i} M_{i}, \Omega^{i+1} M_{i+1}\right) .
$$

The composite $f$ of these maps in $\underline{\operatorname{Hom}}_{k G}(M, N) \cong \operatorname{Ext}_{k G}^{n}\left(M, \Omega^{n} N\right)$ is represented by the Yoneda splice

$$
\begin{aligned}
& 0 \rightarrow \Omega^{n} N \rightarrow \Omega^{n-1} X_{n-1} \oplus(\text { proj }) \rightarrow \ldots \\
& \rightarrow \Omega X_{1} \oplus(\text { proj }) \rightarrow X_{0} \oplus(\text { proj }) \rightarrow M \rightarrow 0
\end{aligned}
$$

of these sequences. This is a pure extension of degree $n$ of $M$ by $\Omega^{n} N$. Conversely, every such pure extension gives rise to a sequence of $n$ phantom maps whose composite corresponds to the resulting extension class.

TheOREM 4.6.2. Suppose that $|k|=\aleph_{t}$. Then the composite of any $t+2$ phantom maps between $k G$-modules factors through a projective module.

P r o o f. By Theorem 2.6.3, $k G$ has pure global dimension at most $t+1$. So for any $k G$-modules $M$ and $N$, $\operatorname{Pext}_{k G}^{t+2}\left(M, \Omega^{n} N\right)=0$. So by Theorem 4.6.1, $\underline{\mathrm{Ph}}_{k G}^{t+2}(M, N)=0$.

THeOREM 4.6.3. Let $k$ be a field of characteristic two and $G$ be a finite group with Klein four Sylow 2-subgroups. If $k$ is countable then the composite of any two phantom maps between $k G$-modules factors through a projective module. If $k$ is uncountable then the composite of any three phantom maps between $k G$-modules factors through a projective module.

Proof. According to Theorem 4.5.3, if $k$ is uncountable then $k G$ has pure global dimension two. It follows from Theorem 4.6.1 that in this case we have $\underline{\operatorname{Ph}}_{k G}^{3}(M, N)=0$ for all $k G$-modules $M$ and $N$. Similarly in the countable case we have $\underline{\mathrm{Ph}}_{k G}^{2}(M, N)=0$ for all $M$ and $N$. 
We may combine this theorem with an idea of Jon Carlson to obtain stronger bounds on the number of phantom maps that must be composed to obtain a map which factors through a projective module. In particular, this number can be smaller than one would expect from the pure global dimension.

Let $H$ be a subgroup of index two in $G$ and let $k$ be a field of characteristic two. Let $M$ and $N$ be $k G$-modules. Then there is a long exact sequence

$$
\begin{aligned}
\ldots \stackrel{x}{\rightarrow} \operatorname{Ext}_{k G}^{n}(M, N) & \stackrel{\operatorname{res}_{G, H}}{\longrightarrow} \operatorname{Ext}_{k H}^{n}(M, N) \\
& \stackrel{\operatorname{Tr}_{H, G}}{\longrightarrow} \operatorname{Ext}_{k G}^{n}(M, N) \stackrel{x}{\rightarrow} \operatorname{Ext}_{k G}^{n+1}(M, N) \stackrel{\stackrel{\operatorname{res}_{G, H}}{\longrightarrow} \ldots}{ }
\end{aligned}
$$

Here, $x$ denotes the element of $\operatorname{Hom}_{\text {Grp }}(G, \mathbb{Z} / 2) \cong H^{1}\left(G, \mathbb{F}_{2}\right) \subseteq H^{1}(G, k)$ corresponding to the homomorphism with kernel $H$, and the maps marked with $x$ are given by cup product with $x$.

THEOREM 4.6.4. Let $k$ be a field of characteristic two, and let $G=$ $\mathbb{Z} / 4 \times \mathbb{Z} / 2$. Then the composite of any six phantom maps between $k G$-modules factors through a projective module.

Pro of. Let $x$ be the element of $H^{1}(G, k)$ corresponding to the subgroup $H=\mathbb{Z} / 2 \times \mathbb{Z} / 2$. Then we have $x^{2}=0$. Since $k H$ has pure global dimension at most two by Theorem 4.5.3, the diagram

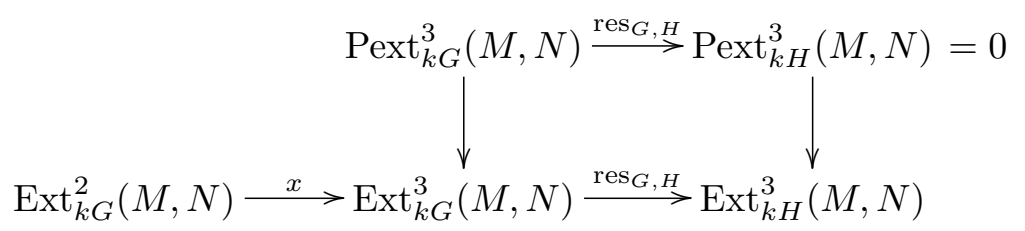

(combined with Theorem 4.6.1) shows that the composite of any three phantom maps between $k G$-modules is divisible by $x$. So the composite of any six phantom maps is divisible by $x^{2}$, and is hence zero.

To apply this method in odd characteristic, instead of making use of the degree one element, one makes use of its Bockstein, using the Quillen-Venkov theorem in the form due to Alperin and Evens (Theorem 5.2.1 of [6]). Serre's theorem on the product of Bocksteins (Theorem 4.7.3 of [6]) ensures that this method may be used whenever $G$ is a p-group which is not elementary abelian. Note that in characteristic two, the Bockstein of a degree one element is its square, so in this case Serre's theorem applies directly to the degree one elements. Of course, one has to start off with good upper bounds in the elementary abelian case, and we only know how to do this in the case of the Klein four group. This gives us the following theorem.

THEOREM 4.6.5. Let $k$ be a field of characteristic two and let $G$ be a finite group of 2-rank at most two. Then there is a number $n(G)$ which depends 
only on $G$ and not on the cardinality of $k$, such that the composite of $n(G)$ phantom maps between $k G$-modules factors through a projective module.

The values of $n(G)$ given by this method are probably not best possible, but here are some of the answers it produces. For the quaternion group, we have $n\left(Q_{8}\right)=3$. This is because the restriction of any phantom map to any subgroup of index two factors through a projective module, and the three elements of $H^{1}\left(Q_{8}, \mathbb{F}_{2}\right)$ have zero product. For the dihedral group we have $n\left(D_{8}\right)=6$, since there are two degree one elements corresponding to Klein four subgroups and having zero product. For the semidihedral group we have $n\left(S D_{16}\right)=18$ because there is a dihedral subgroup of index two corresponding to an element of $H^{1}\left(S D_{16}, \mathbb{F}_{2}\right)$ whose cube is zero. It follows that for the smallest Mathieu group we have $n\left(M_{11}\right)=18$, since it has $S D_{16}$ as a Sylow 2-subgroup.

4.7. Brown representability. In this section, we describe the extent to which the definitions of Hovey, Palmieri and Strickland [21] and of Christensen and Strickland [15] apply to the stable category of $k G$-modules, and we give the translations of the terms given there into representation theory. It is easy to see that $\operatorname{StMod}(k G)$ is an example of a stable homotopy category, in the language of [21]. We shall see below that Brown representability for homology theories does not usually hold, so it is usually not a Brown category.

Spanier-Whitehead duality is just the ordinary $k$-linear duality $M^{*}=$ $\operatorname{Hom}_{k}(M, k)$. Brown-Comenetz duality needs more careful consideration. By analogy with Section 3 of [15], given a $k G$-module $M$ we are required to find a $k G$-module $I M$ such that for every finitely generated $k G$-module $N$,

$$
H^{0}(G, M \otimes N)^{*} \cong \underline{\operatorname{Hom}}_{k G}(N, I M) .
$$

In order to achieve this, we use the form of Tate duality which appears in the construction of almost split sequences. Namely, provided that $N$ is finitely generated (but with no condition on $M$ ), we have a natural isomorphism

$$
\underline{\operatorname{Hom}}_{k G}(N, M)^{*} \cong \underline{\operatorname{Hom}}_{k G}(M, \Omega N) .
$$

This is proved in Section 4.12 of [5] under the assumption that both $M$ and $N$ are finitely generated, but in fact the assumption on $M$ is easily seen to be superfluous. Replacing $M$ by $M \otimes_{k} N$ and $N$ by $k$, we obtain

$$
\begin{aligned}
H^{0}\left(G, M \otimes_{k} N\right)^{*} & =\underline{\operatorname{Hom}}_{k G}(k, M \otimes N)^{*} \\
& \cong \underline{\operatorname{Hom}}_{k G}(M \otimes N, \Omega k) \cong \underline{\operatorname{Hom}}_{k G}\left(N, \Omega M^{*}\right) .
\end{aligned}
$$

We deduce that the Brown-Comenetz dual of $M$ is in fact $\Omega M^{*}$.

A homology theory on a triangulated category $\mathcal{S}$ is an exact covariant functor to an abelian category which takes direct sums to direct sums, to 
the extent that they exist in $\mathcal{S}$. We shall always take for the abelian category the category $\operatorname{Vect}(k)$ of $k$-vector spaces. A map of homology theories is just a natural transformation of functors. We say that two homology theories are equivalent if they are naturally isomorphic as functors.

Given a $k G$-module $M$, we have a homology theory

$$
h_{M}: \operatorname{StMod}(k G) \rightarrow \operatorname{Vect}(k)
$$

given by $h_{M}(N)=\underline{\operatorname{Hom}}_{k G}\left(k, M \otimes_{k} N\right)$. We say that a homology theory $h$ is representable if there exists a $k G$-module $M$ such that $h$ is equivalent to $h_{M}$. We say that a map between representable homology theories is representable if it is induced by a map of modules. We say that Brown representability holds in $\operatorname{StMod}(k G)$ if every homology theory is representable, and furthermore, every map between them is representable.

THEOREM 4.7.1. Let $G$ be a finite group and $k$ be a field of coefficients. Then the following are equivalent.

(i) Brown representability holds for the category $\operatorname{StMod}(k G)$.

(ii) The group algebra $k G$ has pure global dimension zero or one.

(iii) Either $k$ is countable or $G$ has cyclic Sylow p-subgroups.

Proof. The fact that (ii) and (iii) are equivalent follows from Theorem 4.5.3. That (i) implies (ii) follows from Lemma 4.1 of Neeman [26]. That (ii) implies (i) can be obtained from the same paper by combining Proposition 4.11 and Theorem 5.1, or just with the observation following Theorem 5.1. Another proof that (i) implies (ii) is given in Proposition 4.6 of Christensen and Strickland [15].

4.8. Back to topology. Motivated by the connection in the algebraic context between phantom maps and purity, we define a notion of purity for fibrations and investigate its connections with phantom maps.

Definition 4.8.1. We say that a Serre fibration of pointed spaces $\left(F, f_{0}\right)$ $\rightarrow\left(E, e_{0}\right) \rightarrow\left(B, b_{0}\right)$ is a pure fibration if, given any map from a finite $\mathrm{CW}$ complex $\left(A, a_{0}\right)$ to $\left(B, b_{0}\right)$, it lifts to a map to $\left(E, e_{0}\right)$.

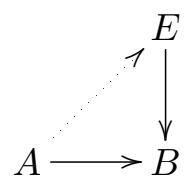

The relationship with phantom maps in the second sense (see Section 4.1) is clear.

Proposition 4.8.2. A map $X \rightarrow Y$ is phantom (with respect to maps from finite complexes) if and only if the corresponding fibration $\Omega Y \rightarrow E \rightarrow$ $X$ obtained by using the path-loop construction is a pure fibration. 
COROLlARY 4.8.3. Let $G$ be a topological group and let $\left(B_{\alpha}, b_{0}\right)$ run over the finite subcomplexes of a pointed $C W$ complex $\left(B, b_{0}\right)$. If $B$ has countably many cells, then the set of principal $G$-bundles over $B$ which are pure fibrations is in natural one-one correspondence with the set $\lim _{\alpha}^{1}\left[\left(B_{\alpha}, b_{0}\right) ;(G, e)\right]$.

Proof. This follows from the classification of principal bundles and the homotopy equivalence $\Omega B G \simeq G$. It is also possible to see the correspondence more directly by choosing sections $B_{\alpha} \rightarrow E_{\alpha}$ for each index $\alpha$ and comparing for pairs of indices.

Proposition 4.8.4. If $\left(F, f_{0}\right) \rightarrow\left(E, e_{0}\right) \rightarrow\left(B, b_{0}\right)$ is a pure fibration of based topological spaces, then the long exact homotopy sequence reduces to short exact sequences

$$
* \rightarrow \pi_{n}\left(F, f_{0}\right) \rightarrow \pi_{n}\left(E, e_{0}\right) \rightarrow \pi_{n}\left(B, b_{0}\right) \rightarrow * .
$$

For $n>1$, this is a pure exact sequence of abelian groups. For $n=1$, it is a pure exact sequence of groups, in the sense that given a finitely presented group $\pi$ and a homomorphism $\pi \rightarrow \pi_{1}\left(B, b_{0}\right)$, there exists a lift to a homomorphism $\pi \rightarrow \pi_{1}\left(E, e_{0}\right)$.

Proof. Since $S^{n}$ is a finite CW complex, every map from $S^{n}$ to $B$ lifts to $E$, and so $\pi_{n}(E) \rightarrow \pi_{n}(B)$ is surjective.

If $\pi$ is a finitely presented group (abelian if $n>1$ ) then a presentation of $\pi$ can be used to build a finite CW complex $\left(A, a_{0}\right)$. The complex $A$ only has cells in dimension $n$, corresponding to the generators of $\pi$, and in dimension $n+1$, with attaching maps specified by the relators. We have $\pi_{n}\left(A, a_{0}\right) \cong \pi$. Given a group homomorphism $\pi \rightarrow \pi_{n}(B)$, choosing representative maps $\left(S^{n}, s_{0}\right) \rightarrow\left(B, b_{0}\right)$ corresponding to the images of the generators of $\pi$ in $\pi_{n}\left(B, b_{0}\right)$, we obtain a map from the $n$-skeleton of $A$ to $B$. The fact that the relators of $\pi$ go to the identity element of $\pi_{n}\left(B, b_{0}\right)$ allows us to extend to a map from $\left(A, a_{0}\right)$ to $\left(B, b_{0}\right)$ inducing the given map $\pi \rightarrow \pi_{n}\left(B, b_{0}\right)$ in homotopy. Since $A$ is a finite $\mathrm{CW}$ complex, we may lift to a map $\left(A, a_{0}\right) \rightarrow\left(E, e_{0}\right)$. This lift induces a lift $\pi \rightarrow \pi_{n}\left(E, e_{0}\right)$ of the original group homomorphism.

EXAMPLE 4.8.5. If $1 \rightarrow N \rightarrow G \rightarrow G / N \rightarrow 1$ is a pure exact sequence of groups then the corresponding fibration of Eilenberg-Mac Lane spaces

$$
K(N, 1) \rightarrow K(G, 1) \rightarrow K(G / N, 1)
$$

is a pure fibration. It follows that if $N=Z$ is central, then the classifying map $K(G / Z, 1) \rightarrow K(Z, 2)$ is phantom.

Similarly, if $0 \rightarrow \pi \rightarrow \pi^{\prime} \rightarrow \pi^{\prime \prime} \rightarrow 0$ is a pure exact sequence of abelian groups then the corresponding fibration of Eilenberg-Mac Lane spaces

$$
K(\pi, n) \rightarrow K\left(\pi^{\prime}, n\right) \rightarrow K\left(\pi^{\prime \prime}, n\right)
$$


is again a pure fibration. In particular, using the path-loop construction, we obtain a phantom map

$$
K\left(\pi^{\prime \prime}, n-1\right) \rightarrow K(\pi, n) .
$$

\section{IDEMPOTENT MODULES AND ENDOMORPHISMS}

Several recent breakthroughs in modular representation theory $[7,9,10$, 11] have depended on Rickard's construction [30] of families of idempotent infinite-dimensional modules. Let $G$ be a finite group and $k$ an algebraically closed field of coefficients. With each collection $\mathcal{V}$ of closed homogeneous irreducible subvarieties of the cohomology variety $V_{G}$, closed under specialization (i.e., if $V$ is in $\mathcal{V}$ and $W \subseteq V$ then $W$ is also in $\mathcal{V}$ ), Rickard [30] associates a distinguished triangle in $\operatorname{StMod}(k G)$ :

$$
E(\mathcal{V}) \stackrel{\varepsilon}{\longrightarrow} k \stackrel{\eta}{\longrightarrow} F(\mathcal{V}) \rightarrow \Omega^{-1} E(\mathcal{V}) .
$$

This triangle is characterized in terms of varieties for modules as follows. Let $\mathcal{C}(\mathcal{V})$ be the thick subcategory of $\operatorname{stmod}(k G)$ consisting of $k G$-modules whose variety $V_{G}(M) \subseteq V_{G}$ is a finite union of elements of $\mathcal{V}$. Then $E(\mathcal{V})$ is a filtered colimit of modules in $\mathcal{C}(\mathcal{V})$, and $F(\mathcal{V})$ is $\mathcal{C}(\mathcal{V})$-local in the sense that if $X$ is an object in $\mathcal{C}(\mathcal{V})$ then $\underline{\operatorname{Hom}}_{k G}(X, F(\mathcal{V}))=0$. Any other distinguished triangle with the second term isomorphic to $k$ and first and third terms with these properties is isomorphic to the above triangle. We refer to this statement as Rickard's characterization of triangles for idempotent modules. (The modules $E(\mathcal{V})$ and $F(\mathcal{V})$ are idempotent in the sense that they are stably isomorphic to their tensor squares.) In case $\mathcal{V}$ consists of all the closed homogeneous irreducible subvarieties of a given closed homogeneous subvariety $V \subseteq V_{G}$, we write $E(V)$ and $F(V)$ for the modules $E(\mathcal{V})$ and $F(\mathcal{V})$. In this section we investigate the endomorphism rings of these modules, and provide some evidence for our conjecture that the modules $E(V)$ are pure injective. We prove this conjecture in the special case where $V_{G}$ is two-dimensional and $V$ is one-dimensional.

5.1. The four group. We begin with an example where the structure of the pure injective modules is explicitly known. Let $k$ be an algebraically closed field of characteristic two and let $G=\mathbb{Z} / 2 \times \mathbb{Z} / 2$ be the Klein four group.

The canonical functors between the category of Kronecker modules and the category of $k G$-modules preserve pure injective modules, by Corollary 2.3.7. Therefore the classification of pure injective $k G$-modules can be read off from the classification of pure injective Kronecker modules given in Chapter 8 of Jensen and Lenzing [22]. They attribute the classification to Geigle. 
THEOREM 5.1.1. Every pure injective $k G$-module is the pure injective envelope of a direct sum of indecomposable pure injective $k G$-modules. Every indecomposable pure injective $k G$-module is isomorphic to a unique module from the following list:

(i) The finitely generated $k G$-modules.

(ii) The modules $E(V)$, where $V$ is a line through the origin in $V_{G}=$ $\mathbb{A}^{2}(k)$.

(iii) The $k$-duals $E(V)^{*}$ to the modules of (ii). These are called the Prüfer modules.

(iv) The module $F(\mathcal{U})$, where $\mathcal{U}$ is the collection of all lines through the origin in $V_{G}$.

Let $\zeta$ be a homogeneous irreducible element of $H^{*}(G, k)$, let $V$ be the line through the origin in $V_{G}=\mathbb{A}^{2}(k)$ it defines, and let $(\zeta)$ denote the homogeneous prime ideal it generates in $H^{*}(G, k)$. Then it is easy to see from the construction that the $k G$-modules $E(V)$ and $E(V)^{*}$ correspond respectively to the Prüfer module $S_{(\zeta)}^{(\infty)}$ and the $(\zeta)$-adic Kronecker module $J_{(\zeta)}$ in the notation of Jensen and Lenzing in Chapter 8 of [22]. That the modules $E(V)$ are pure injective is a special case of what will be discussed in Section 5.3. The modules $E(V)^{*}$ are pure injective by Theorem 2.3.3. For the case (iv), we have the following:

THEOREM 5.1.2. The $k G$-module $L$ corresponding to the Kronecker module

$$
\Phi=k(X) \stackrel{1}{\underset{X}{\longrightarrow}} \geq k(X)
$$

is pure injective and is a representative in $\operatorname{Mod}(k G)$ of $F(\mathcal{U})$. Moreover, $L$ is isomorphic to $\left(L_{\zeta}\right) \downarrow_{k G}$, where $\zeta$ is the generic cohomology element

$$
\zeta=u_{1}+X u_{2} \in H^{*}(G, k(X))=k(X)\left[u_{1}, u_{2}\right],
$$

and $L_{\zeta}$ is the kernel of the representative map $\widehat{\zeta}: \Omega(k(X)) \rightarrow k(X)$.

Proof. Let $g_{1}$ and $g_{2}$ be generators for $G$. As a $k$-linear space, $L=$ $L_{0} \oplus L_{1}$, where $L_{0}$ and $L_{1}$ are copies of $k(X)$. The subspace $L_{1}$ is the space of $G$-invariants, and $g_{1}-1$ and $g_{2}-1$ send $L_{0}$ isomorphically onto $L_{1}$ via the identity and via multiplication by $X$ respectively. The module $L$ also admits the ring structure of $k(X)[\alpha] /\left(\alpha^{2}\right)$ with $L_{0}$ and $L_{1}$ equal to the multiples of 1 and $\alpha$ respectively, and $L$ is then a $k E$ - $L$-bimodule. The ring $L$ is self-injective. So the isomorphism

$$
{ }_{k G} L_{L} \otimes_{L} L L \cong{ }_{k G} L
$$

together with Corollary 2.3.7 show that $L$ is pure injective. 
To show that $L$ represents $F(\mathcal{U})$, we use Rickard's characterization of distinguished triangles for idempotent modules. Let $\eta: k \rightarrow L_{1} \subset L$ be the $k G$-homomorphism sending 1 to 1 .

(i) Let $(0,0) \neq(\lambda, \mu) \in \mathbb{A}^{2}(k)$. Set $a=1+\lambda\left(g_{1}-1\right)+\mu\left(g_{2}-1\right) \in k G$. Then $a-1$ sends $L_{0}$ to $L_{1}$ via multiplication by $\lambda+\mu X$; this is an isomorphism. So $L \downarrow_{k\langle a\rangle}$ is free.

(ii) Let $t$ be an indeterminate. Set $a=1+\left(g_{1}-1\right)+t\left(g_{2}-1\right) \in k(t) G$. Let $\psi: k(t) \otimes k(X) \rightarrow k(t), f(t) \otimes g(X) \mapsto f(t) g(1 / t)$. Then $a-1$ sends $k(t) \otimes L_{0} \cong k(t) \otimes k(X)$ into Ker $\psi$ by multiplication by $1 \otimes 1+t \otimes X$. It is an elementary exercise to show that this is a $k(t)$-isomorphism. So the $k(t)$-linear subspace $\widehat{L}=\left(k(t) \otimes L_{0}\right) \oplus \operatorname{Ker} \psi$ of $k(t) \otimes L$ is a free submodule of $(k(t) \otimes L) \downarrow_{k(t)\langle a\rangle}$. Now the monomorphism $k(t) \otimes \eta: k(t) \rightarrow k(t) \otimes L_{1} \cong$ $k(t) \otimes k(X)$ splits with splitting epimorphism $\psi$. So $(k(t) \otimes L) \downarrow_{k(t)\langle a\rangle} \cong$ $k(t) \oplus \widehat{L}$.

By [10], the rank variety of $L$ consists of $V_{G}$ only and that of Coker $(\eta$ : $k \rightarrow L)$ is $\mathcal{U}$. Now by Rickard's characterization of distinguished triangles for idempotent modules, $L$ is a representative in $\operatorname{Mod}(k G)$ of $F(\mathcal{U})$.

That $L$ is isomorphic to $\left(L_{\zeta}\right) \downarrow_{k G}$ is left as an exercise.

This theorem has a generalization to odd characteristic (Theorem 5.4.6).

5.2. Commutativity. Since $E(\mathcal{V})$ and $F(\mathcal{V})$ should really be considered as objects in the stable category, it makes sense to look at their endomorphism rings there - namely, the quotient $\underline{\operatorname{End}}_{k G}(E(\mathcal{V}))$ of $\operatorname{End}_{k G}(E(\mathcal{V}))$ by those endomorphisms which factor through a projective module (and similarly for $F(\mathcal{V}))$.

LEMmA 5.2.1. (i) The stable isomorphisms $\varepsilon \otimes 1$ and $1 \otimes \varepsilon$ from $E(\mathcal{V}) \otimes$ $E(\mathcal{V})$ to $E(\mathcal{V})$ are equal in $\operatorname{StMod}(k G)$ (i.e., they differ in $\operatorname{Mod}(k G)$ by a map which factors through a projective module).

(ii) The stable isomorphisms $\eta \otimes 1$ and $1 \otimes \eta$ from $F(\mathcal{V})$ to $F(\mathcal{V}) \otimes F(\mathcal{V})$ are equal in $\operatorname{StMod}(k G)$.

Proof. (i) We have $\varepsilon \circ(\varepsilon \otimes 1-1 \otimes \varepsilon)=\varepsilon \otimes \varepsilon-\varepsilon \otimes \varepsilon=0$. So $\varepsilon \otimes 1-$ $1 \otimes \varepsilon$ factors through the connecting homomorphism $\Omega F(\mathcal{V}) \rightarrow E(\mathcal{V})$. Since $\Omega F(\mathcal{V})$ is $\mathcal{C}(\mathcal{V})$-local, every homomorphism from $E(\mathcal{V}) \otimes E(\mathcal{V})$ to $\Omega F(\mathcal{V})$ factors through a projective module, and hence so does $\varepsilon \otimes 1-1 \otimes \varepsilon$.

(ii) The proof is dual.

TheOrem 5.2.2. The rings $\underline{\operatorname{End}}_{k G}(E(\mathcal{V}))$ and $\underline{\operatorname{End}}_{k G}(F(\mathcal{V}))$ are commutative.

Pr o of. We prove that the ring $\operatorname{End}_{k G}(E(\mathcal{V}))$ is commutative. The proof for $\underline{\operatorname{End}}_{k G}(F(\mathcal{V}))$ is dual. Denote by $\widehat{\varepsilon}: E(\mathcal{V}) \otimes E(\mathcal{V}) \rightarrow E(\mathcal{V})$ the map 
$\varepsilon \otimes 1=1 \otimes \varepsilon$ in $\operatorname{StMod}(k G)$ of Lemma 5.2.1. If $\alpha$ and $\beta$ are endomorphisms of $E(\mathcal{V})$ then consider the diagram

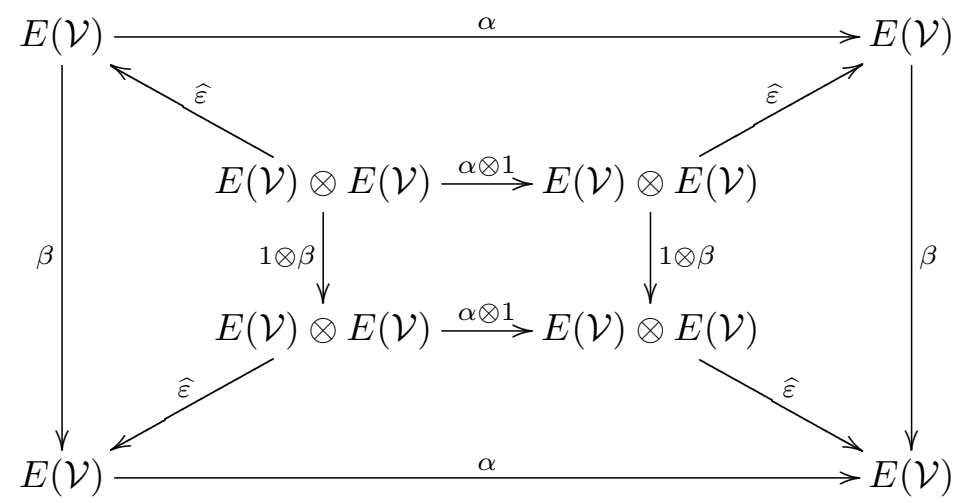

in $\operatorname{StMod}(k G)$. The inside square commutes. To see that the parts involving $\widehat{\varepsilon}$ commute, interpret $\widehat{\varepsilon}$ as $\varepsilon \otimes 1$ for the left and right parts and as $1 \otimes \varepsilon$ for the top and bottom parts. It follows that the outside square commutes.

For objects $M$ and $N$ in the triangulated category $\operatorname{StMod}(k G)$, we should really consider the morphisms from $M$ to $N$ to be a graded object consisting of the Tate cohomology groups

$$
\widehat{\operatorname{Ext}}_{k G}^{i}(M, N)=\underline{\operatorname{Hom}}_{k G}\left(\Omega^{i} M, N\right) .
$$

The graded commutativity of the Tate cohomology ring $\widehat{H}^{*}(G, k)$ can be combined with the above theorem as follows.

TheOREM 5.2.3. The graded endomorphism rings

$$
\widehat{\operatorname{Ext}}_{k G}^{*}(E(\mathcal{V}), E(\mathcal{V})) \quad \text { and } \quad \widehat{\operatorname{Ext}}_{k G}^{*}(F(\mathcal{V}), F(\mathcal{V}))
$$

are graded commutative.

Proof. We begin with the following interpretation of the graded commutativity of Tate cohomology. For $n \geq 0$, the tensor $n$th power of $\Omega k$ is isomorphic to $\Omega^{n} k$ plus projective. The $k$-vector space dual of $\Omega k$ is $\Omega^{-1} k$, and the tensor $n$th power of $\Omega^{-1} k$ is $\Omega^{-n} k$ plus projective. With these identifications, the twist map $\Omega^{m} k \otimes \Omega^{n} k \rightarrow \Omega^{n} k \otimes \Omega^{m} k$ sending $x \otimes y$ to $y \otimes x$ differs from $(-1)^{m n}$ times the identity map by a map which factors through a projective module. Now for any module $M$, we can identify $\Omega^{n} M$ with $\Omega^{n} k \otimes M$ in the stable category.

Each of the inside parts of the following diagram commutes in the stable category, and hence so does the outside part: 


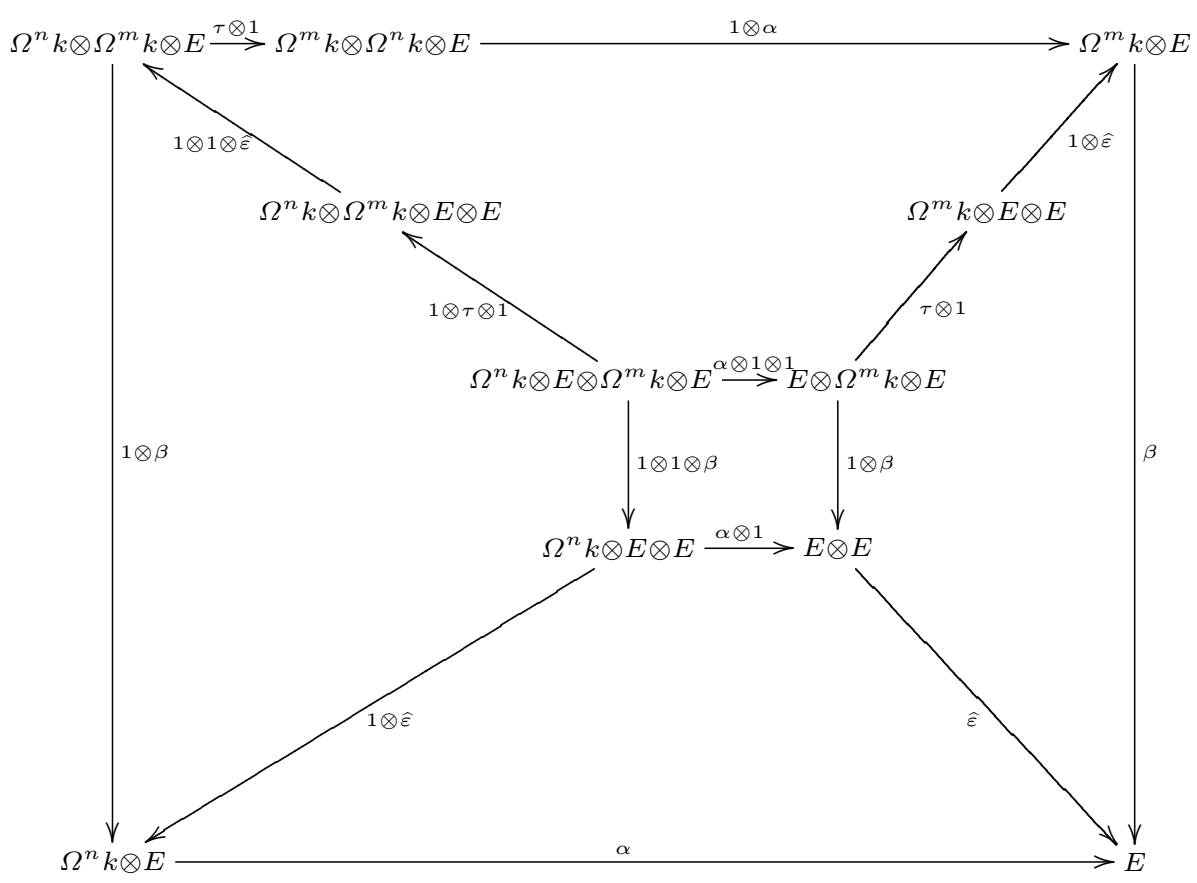

To check that the diagram commutes, interpret $\widehat{\varepsilon}$ as $1 \otimes \varepsilon$ for the purposes of checking that the top and bottom parts commute and as $\varepsilon \otimes 1$ for the purposes of checking that the left and right parts commute.

5.3. Pure injectivity. In this section, we prove the following theorem.

TheOrem 5.3.1. Let $G$ be a finite group of p-rank two and let $k$ be an algebraically closed field of characteristic p (so that by Quillen's dimension theorem, $V_{G}$ is two-dimensional). If $V$ is a closed homogeneous subvariety of $V_{G}$, then $E(V)$ is pure injective.

Pr o of. The idea of the proof of this theorem is to apply Corollary 2.3.7 to a suitable functor from $\operatorname{Mod}(k[[x]])$ to $\operatorname{Mod}(k G)$. This functor is given by tensoring with a $k G-k[[x]]$-bimodule $Y$ which we construct below. This bimodule needs to be finitely generated as a $k[[x]]$-module so that direct products are preserved; direct sums are automatically preserved. We construct the bimodule as the limit of an inverse system of $k G$-modules, and the action of $k[[x]]$ comes through the construction of certain maps of inverse systems. Part of the difficulty is that we need to perform these constructions in the module category, not just in the stable category.

We may suppose that $V$ has dimension one, and can be written as the set of zeros of a homogeneous element $\zeta \in H^{*}(G, k)$, and that we can choose another homogeneous element $\eta \in H^{*}(G, k)$ which takes nonzero value on $V$. 
By replacing these elements by suitable powers if necessary, we may assume that they have the same degree, $\zeta, \eta \in H^{n}(G, k)$.

If $\alpha$ is a nonzero homogeneous element of $H^{*}(G, k)$ of degree $m$, we let $L_{\alpha}$ denote the kernel of the representative map $\widehat{\alpha}: \Omega^{m} k \rightarrow k$; see Section 5.9 of [6] for more on these modules. $\eta$ generates the periodicity of $L_{\zeta^{i}}$ in the sense of Section 5.10 of [6]. Tensoring $L_{\zeta^{i}}($ for $i>0$ ) with the short exact sequence

$$
0 \rightarrow L_{\eta} \rightarrow \Omega^{n} k \stackrel{\widehat{\eta}}{\longrightarrow} k \rightarrow 0,
$$

we see that $\Omega^{n} L_{\zeta^{i}} \cong L_{\zeta^{i}}$.

We have a filtered system of distinguished triangles in $\operatorname{stmod}(k G)$ :

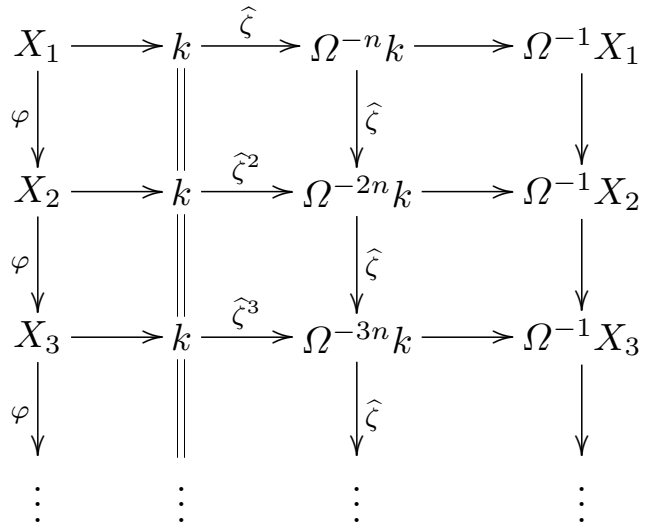

whose colimit is the triangle

$$
E(V) \rightarrow k \rightarrow F(V) \rightarrow \Omega^{-1} E(V) .
$$

Here,

$$
\left.\left.X_{i} \cong \Omega^{-n i} L_{\zeta^{i}} \oplus \text { (projective }\right) \cong L_{\zeta^{i}} \oplus \text { (projective }\right),
$$

the last isomorphism being induced by $\eta^{i}$.

Applying the octahedral axiom for $\operatorname{stmod}(k G)$ to the diagram

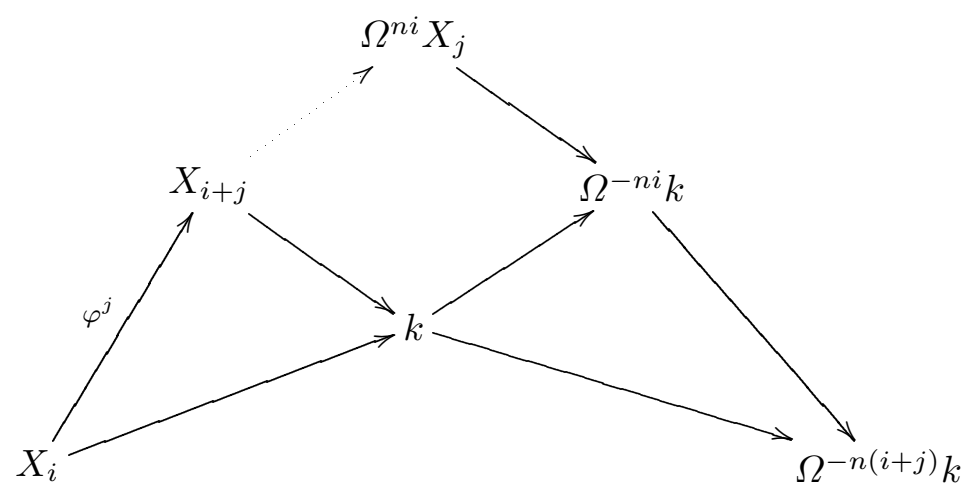

we see that there are natural distinguished triangles 


$$
X_{i} \stackrel{\varphi^{j}}{\rightarrow} X_{i+j} \rightarrow \Omega^{-n i} X_{j} \rightarrow \Omega^{-1} X_{i}
$$

induced by the map from $X_{i}$ to $X_{i+j}$ in the above system $\left(^{1}\right)$, and $\eta^{i}$ then provides a stable isomorphism between the term $\Omega^{-n i} X_{j}$ and $X_{j}$, so that we have distinguished triangles

$$
X_{i} \stackrel{\varphi^{j}}{\longrightarrow} X_{i+j} \rightarrow X_{j} \rightarrow \Omega^{-1} X_{i} .
$$

The case $i=1$ gives us maps $\psi: X_{j+1} \rightarrow X_{j}$.

Also, applying the octahedral axiom for $\operatorname{stmod}(k G)$ to the diagram

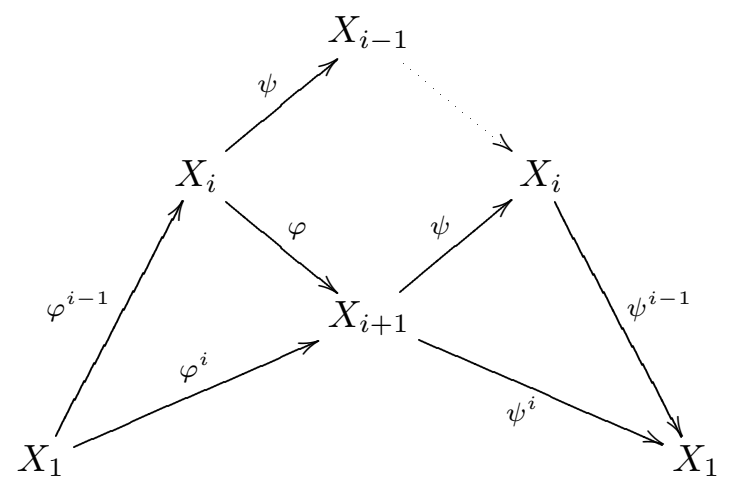

constructs the dotted arrow, which must then be equal to $\varphi$ since it is determined by $\psi^{i-1}$. So the squares

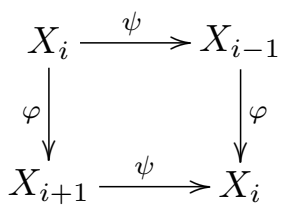

commute in $\operatorname{stmod}(k G)$.

We claim that by adding or removing projective summands to or from $X_{i}$, and correctly choosing the representative maps $\varphi: X_{i} \rightarrow X_{i+1}$ and $\psi: X_{i+1} \rightarrow X_{i}$ in $\bmod (k G)$, we can obtain that the sequences

$$
0 \rightarrow X_{i} \stackrel{\varphi}{\longrightarrow} X_{i+1} \stackrel{\psi^{i}}{\longrightarrow} X_{1} \rightarrow 0
$$

are exact and the squares

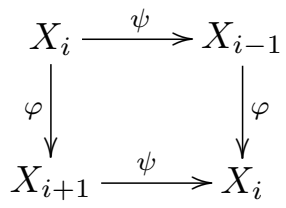

$\left.{ }^{1}\right)$ In general, two maps in a morphism of triangles do not uniquely determine the third. However, we are using the fact that if one the two maps is an isomorphism, then the third is uniquely determined. 
commute in $\bmod (k G)$. We prove this by induction. First choose modules $X_{1}$ and $X_{2}$, and maps $\varphi: X_{1} \rightarrow X_{2}$ and $\psi: X_{2} \rightarrow X_{1}$ such that the sequence

$$
0 \rightarrow X_{1} \stackrel{\varphi}{\longrightarrow} X_{2} \stackrel{\psi}{\longrightarrow} X_{1} \rightarrow 0
$$

is exact. Then assume for induction that the sequence

$$
0 \rightarrow X_{i} \stackrel{\varphi}{\longrightarrow} X_{i+1} \stackrel{\psi^{i}}{\longrightarrow} X_{1} \rightarrow 0
$$

is exact. We enlarge the module $X_{i+2}$ with a projective summand so that in the diagram

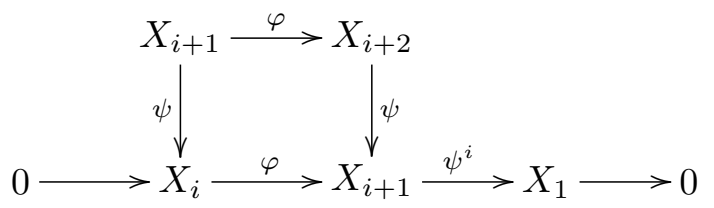

the square is commutative $(\operatorname{in} \bmod (k G))$ and the maps $\varphi: X_{i+1} \rightarrow X_{i+2}$ and $\psi: X_{i+2} \rightarrow X_{i+1}$ are injective and surjective respectively. Then we have the distinguished triangle

$$
X_{i+1} \stackrel{\varphi}{\longrightarrow} X_{i+2} \stackrel{\psi^{i+1}}{\longrightarrow} X_{1}
$$

with the map $\varphi: X_{i+1} \rightarrow X_{i+2}$ injective, the map $\psi^{i+1}: X_{i+2} \longrightarrow X_{1}$ surjective, and $\psi^{i+1} \varphi=0$. It follows that we can remove a projective summand from the module $X_{i+2}$ so as to turn the distinguished triangle into an exact sequence

$$
0 \rightarrow X_{i+1} \stackrel{\varphi}{\longrightarrow} X_{i+2} \stackrel{\psi^{i+1}}{\longrightarrow} X_{1} \rightarrow 0 .
$$

And of course the square remains commutative. So the claim is proved. Now applying the $3 \times 3$ Lemma on the diagram

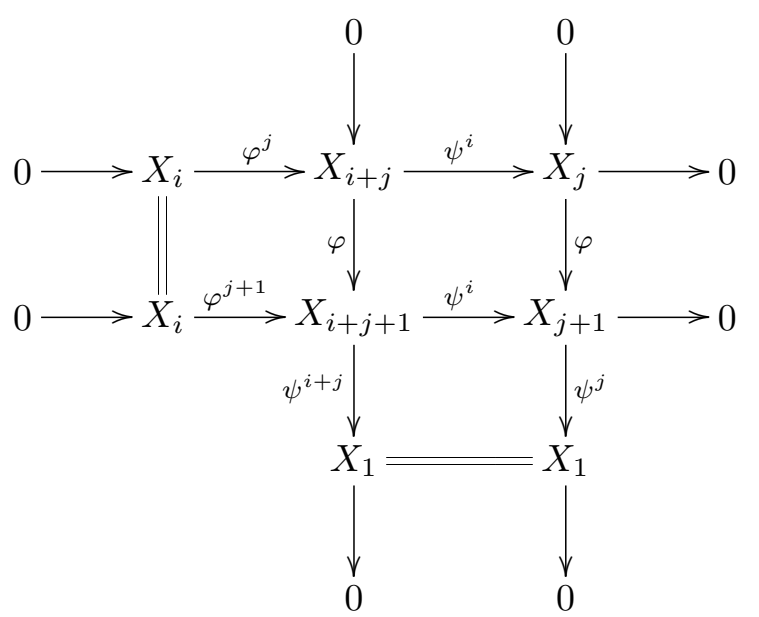


we deduce by induction on $j$ that the sequences

$$
0 \rightarrow X_{i} \stackrel{\varphi^{j}}{\longrightarrow} X_{i+j} \stackrel{\psi^{i}}{\longrightarrow} X_{j} \rightarrow 0
$$

are exact.

We have $E(V)=\lim X_{i}$. Let $Y=\lim X_{i}$ regarded as a $k G$-submodule

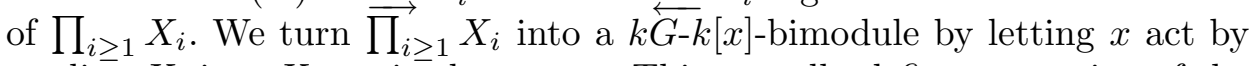
sending $X_{i}$ into $X_{i+1}$ via the map $\varphi$. This actually defines an action of the ring $k[[x]]$ of formal power series. Indeed, given $y \in \prod_{i \geq 1} X_{i}$, the coordinate of index $i$ of $x^{j} \cdot y$ is zero for $j \geq i$. Now by the commutative squares above, $Y$ is a $k G-k[[x]]$-submodule of $\prod_{i \geq 1} X_{i}$. Using the exactness of the sequences

$$
0 \rightarrow X_{i} \stackrel{\varphi^{j}}{\longrightarrow} X_{i+j} \stackrel{\psi^{i}}{\longrightarrow} X_{j} \rightarrow 0
$$

and the commutativity of the squares, one can see that the natural map $Y \rightarrow X_{i}$ is surjective with kernel $x^{i} Y$. So we have the direct sequence of $k G$-isomorphims

$$
Y \otimes_{k[[x]]} k[x] /\left(x^{i}\right) \stackrel{\cong}{\longrightarrow} Y / x^{i} Y \stackrel{\cong}{\longrightarrow} X_{i},
$$

with the map $Y / x^{i} Y \rightarrow Y / x^{i+1} Y$ induced by the action of $x$ on $Y$. Applying $\longrightarrow$ lim, and using the fact that as $k G$-modules

$$
\varliminf_{\longrightarrow} k[x] /\left(x^{i}\right) \cong k\left[x^{-1}\right],
$$

we get

$$
Y \otimes_{k[[x]]} k\left[x^{-1}\right] \cong E(V) .
$$

Since $Y / x Y$ is finite-dimensional (being isomorphic to $X_{1}$ ) and since the ring $k[[x]]$ is local with maximal ideal $(x), Y$ is finitely $k[[x]]$-generated, by Nakayama's lemma. On the other hand $k\left[x^{-1}\right]$ is injective as a $k[[x]]$-module, being divisible over the PID $k[[x]]$. (In fact $k\left[x^{-1}\right]$ is the injective envelope of the trivial $k[[x]]$-module $k$.) So by Corollary 2.3.7, $E(V)$ is pure injective.

In [23], Krause studies the so-called generalized tubes and shows how to these correspond certain pure injective modules. Our proof shows that $E(V)$ is such a module.

COROLlary 5.3.2. In the situation of the above theorem, there are no nontrivial phantom maps to $E(V)$.

Proof. This follows from the above theorem together with Theorem 4.2.5.

5.4. All proper subvarieties. Let $E$ be a finite elementary abelian $p$-group of order $p^{r}$ with $r \geq 2$, and let $k$ be an algebraically closed field of characteristic $p$. Let $\mathcal{U}$ denote the collection of all proper closed homogeneous irreducible subvarieties of the cohomology variety $V_{E} \cong \mathbb{A}^{r}(k)$. In 
this section, we examine the module $F(\mathcal{U})$. The cohomology ring of $E$ is as follows:

$$
H^{*}(E, k)= \begin{cases}k\left[u_{1}, \ldots, u_{r}\right], & p=2, \\ \Lambda\left(v_{1}, \ldots, v_{r}\right) \otimes_{k} k\left[u_{1}, \ldots, u_{r}\right], & p>2 .\end{cases}
$$

Here, the exterior generators $v_{i}$ have degree one, and the Bockstein of $v_{i}$ is $u_{i}$ of degree two. It follows from the work of Carlson, Donovan and Wheeler in [12] and that of Carlson and Wheeler in [13] that as a ring and as a module over $H^{*}(E, k)$, we have

$$
\widehat{H}^{*}(E, F(\mathcal{U})) \cong \begin{cases}k\left(u_{1}, \ldots, u_{r}\right)_{\mathrm{gr}}, & p=2, \\ \Lambda\left(v_{1}, \ldots, v_{r}\right) \otimes_{k} k\left(u_{1}, \ldots, u_{r}\right)_{\mathrm{gr}}, & p>2 .\end{cases}
$$

Here, the subscript "gr" is used to denote the graded ring of fractions, namely the subring of the full function field in which only the nonzero homogeneous elements have been inverted. In the sequel of this section, we assume that $k$ has cardinality $\aleph_{t}$.

TheOREM 5.4.1. As a graded module over $H^{*}(E, k), \widehat{H}^{*}(E, F(\mathcal{U}))$ is flat and has projective dimension $\min (r, t+1)$.

Proof. For $p=2$, this follows directly from Theorem 2.7.4. For $p>2$, everything has to be tensored with the exterior algebra, which does not change the projective dimension. The latter statement is an application of the lemma below.

We actually need the projective dimension of $\widehat{H}^{*}(E, F(\mathcal{U}))$ over $\widehat{H}^{*}(E, k)$ in order to determine the pure projective dimension of $F(\mathcal{U})$ and assert the existence of a pure projective resolution of $F(\mathcal{U})$ of minimal length and where the modules are of a particularly interesting nature (Theorem 5.4.4). The following lemma will help toward this goal.

Lemma 5.4.2. Let $S$ be a ring. Assume that $S=I \oplus R$ where $I$ is a two-sided ideal and $R$ is a subring. Let $X$ be a flat $S$-module such that $I \otimes_{R} X=0$. Then ( $X$ is flat as an $R$-module and) the projective dimensions of $X$ as an $R$-module and as an $S$-module are equal. The result remains true if one is concerned with graded rings and modules.

Proof. First, note that if $P$ is a projective $S$-module, then $P / I P$ is a projective $R$-module. And if $Q$ is a projective $R$-module, then $S \otimes_{R} Q$ is a projective $S$-module. To see that $X$ is flat as an $R$-module, note that every $R$-module can be regarded as an $S$-module on which $I$ acts as zero, and that under this assumption the tensor product of two $R$-modules is isomorphic to their tensor product as $S$-modules. Let $P_{*} \rightarrow X$ be a projective resolution of $X$ as an $S$-module. Applying $(S / I) \otimes_{S}-$, and knowing that the resolution is pure ( $X$ being flat, see Section 2.7), we get for the $R$-module $X$ the projective resolution $P_{*} / I P_{*} \rightarrow X$. 
For the converse, note that $S \otimes_{R} X \cong X$ as $S$-modules, and apply $S \otimes_{R}-$ to a projective resolution of the $R$-module $X$.

THEOREM 5.4.3. As a graded module over $\widehat{H}^{*}(E, k), \widehat{H}^{*}(E, F(\mathcal{U}))$ is flat and has projective dimension $\min (r, t+1)$.

Proof. The work of Benson and Carlson in [8] gives information on the ring structure of the Tate cohomology ring $\widehat{H}^{*}(E, k)$. Since $r \geq 2$, we have

$$
\widehat{H}^{*}(E, k)=H^{-}(E, k) \oplus H^{*}(E, k)
$$

where $H^{-}(E, k)$, the negative Tate cohomology, is a square-zero two-sided ideal, and the ordinary cohomology $H^{*}(E, k)$ is a subring. We have

$$
\left(H^{-}(E, k) \cdot H^{*}(E, k)\right) \cap H^{*}(E, k)=0 .
$$

Since $\widehat{H}^{*}(E, F(\mathcal{U}))$ is divisible by the homogeneous elements of $H^{*}(E, k)$, we have $H^{-}(E, k) \otimes_{H^{*}(E, k)} \widehat{H}^{*}(E, F(\mathcal{U}))=0$. The ring $\widehat{H}^{*}(E, F(\mathcal{U}))$ is obtained from $H^{*}(E, k)$ by localizing at the multiplicative subset of homogeneous elements in the polynomial subring. Localizing $\widehat{H}^{*}(E, k)$ at this same multiplicative set again gives $\widehat{H}^{*}(E, F(\mathcal{U}))$. So $\widehat{H}^{*}(E, F(\mathcal{U}))$ is $\widehat{H}^{*}(E, k)$-flat. Now combine the above lemma and Theorem 4.5.1 to get the result.

TheOREM 5.4.4. As a kE-module, $F(\mathcal{U})$ has pure projective dimension $\min (r, t+1)$ and has a pure projective resolution of minimal length where the pure projective modules are direct sums of $\Omega$-translates of $k$ and free modules.

Proof. The first statement is obtained by combining Corollary 4.4.4 and Theorem 5.4.3. The latter statement follows from Corollary 4.4.3.

Next, we discuss the following conjecture.

Conjecture 5.4.5. $F(\mathcal{U})$ is a pure injective $k E$-module.

In case $E$ is the Klein four group and $p=2$, this statement is in Theorem 5.1.2. We prove the conjecture in case $E=\left\langle g_{1}, g_{2}\right\rangle$ is elementary abelian of rank two and $p>2$.

THEOREM 5.4.6. If $E$ has rank two then $F(\mathcal{U})$ is a pure injective $k E$ module.

Proof. We assume $p>2$. This case is similar to the case $p=2$, but more complicated. We set

$$
R=k(X)[\alpha, \beta, \gamma] /\left(\alpha \gamma-\beta^{p-1}, \beta \gamma-X \alpha^{p-1}, \alpha \beta \gamma, \gamma^{2}-X \alpha^{p-2} \beta^{p-2}\right) .
$$

In this commutative ring, we have $\alpha^{p}=\alpha\left(X^{-1} \beta \gamma\right)=0, \beta^{p}=\beta(\alpha \gamma)=0$, and $\alpha^{p-1} \beta^{p-1}=\left(X^{-1} \beta \gamma\right)(\alpha \gamma)=0$. It is easy to check from this that $R$ has dimension $p^{2}$ as a $k(X)$-module, with basis consisting of the elements $\alpha^{i} \beta^{j}$ $(0 \leq i, j \leq p-1$, but not both equal to $p-1)$, and $\gamma$. 
The ring $R$ is not self-injective, but the injective hull of $k(X)$ as an $R$-module is the module ${ }_{R} I$ with generators $a$ and $b$, and relations $\beta a=\alpha b$, $\gamma a=\beta^{p-2} b$ and $\gamma b=X \alpha^{p-2} a$. It is easy to check that ${ }_{R} I$ also has dimension $p^{2}$ as a $k(X)$-module.

We regard $R$ and $I$ as infinitely generated $k E$-modules with $g_{1}$ acting as multiplication by $1+\alpha$ and with $g_{2}$ acting as multiplication by $1+\beta$. This makes sense because $\alpha$ and $\beta$ commute and their $p$ th powers are zero. Thus we have a $k E$ - $R$-bimodule ${ }_{k E} R_{R}$ and an infinitely generated $k E$-module ${ }_{k E} I \cong{ }_{k E} R_{R} \otimes_{R} R$. We have a map of $k E$-modules $k \rightarrow{ }_{k E} I$ given by

$$
1 \mapsto \alpha \gamma b=\beta^{p-1} b=\beta \gamma a=X \alpha^{p-1} a,
$$

and we check that

(i) when restricted to any cyclic shifted subgroup

$$
\left\langle 1+\lambda\left(g_{1}-1\right)+\mu\left(g_{2}-1\right)\right\rangle
$$

$\left((\lambda, \mu) \neq(0,0) \in \mathbb{A}^{2}(k)\right)$ of $k E,{ }_{k E} I$ is free, while

(ii) when restricted to the generic cyclic shifted subgroup

$$
\left\langle 1+\left(g_{1}-1\right)+t\left(g_{2}-1\right)\right\rangle
$$

of $k(t) E$ ( $t$ an indeterminate), the cokernel of $k(t) \rightarrow k(t) \otimes_{k}{ }_{k} I$ is free.

It then follows that ${ }_{k E} I$ is isomorphic to $F(\mathcal{U})$ in $\operatorname{StMod}(k E)$. As before, we have a functor $\operatorname{Mod}(R) \rightarrow \operatorname{Mod}(k E)$ given by ${ }_{k E} R_{R} \otimes_{R}-$. This takes direct sums to direct sums, and direct products to direct products. By Corollary 2.3.7, the image

$$
{ }_{k E} R_{R} \otimes_{R} I \cong{ }_{k E} I \cong F(\mathcal{U})
$$

of the injective $R$-module ${ }_{R} I$ is a pure injective $k E$-module.

5.5. Structure of $\underline{\operatorname{End}}_{k G}(E(\mathcal{V}))$. We reduce the study of $\operatorname{End}_{k G}(E(\mathcal{V}))$ to that of $\underline{\operatorname{Hom}}_{k G}(\Omega F(\mathcal{V}), k)$. Carlson and Wheeler [13] have shown how to express $F(\mathcal{V})$ as a filtered colimit of the duals of the modules $U\left(\zeta_{1}, \ldots, \zeta_{s}\right)$ as $\left(\zeta_{1}, \ldots, \zeta_{s}\right)$ runs over the sequences such that $V\left(\zeta_{1}, \ldots, \zeta_{s}\right) \in \mathcal{V}$. This allows us to write $\underline{\operatorname{Hom}}_{k G}(\Omega F(\mathcal{V}), k)$ as an inverse limit of the cohomology groups $H^{1}\left(G, U\left(\zeta_{1}, \ldots, \zeta_{s}\right)\right)$. We describe this inverse limit explicitly in the case where $\mathcal{V}$ is a finite collection of hypersurfaces, and interpret the multiplication in terms of Massey products in Tate cohomology.

LEMMA 5.5.1. There are natural isomorphisms

(i) $\underline{\operatorname{End}}_{k G}(E(\mathcal{V})) \cong \underline{\operatorname{Hom}}_{k G}(E(\mathcal{V}), k)$, and

(ii) $\underline{\operatorname{End}}_{k G}(F(\mathcal{V})) \cong \underline{\operatorname{Hom}}_{k G}(k, F(\mathcal{V}))$. 
Proof. This follows from the long exact sequences associated with the triangle

$$
E(\mathcal{V}) \rightarrow k \rightarrow F(\mathcal{V}) \rightarrow \Omega^{-1} E(\mathcal{V})
$$

and the fact that $\underline{\operatorname{Hom}}_{k G}(E(\mathcal{V}), F(\mathcal{V}))=0$.

TheOREM 5.5.2. There is an exact sequence

$$
0 \rightarrow k \rightarrow \underline{\operatorname{End}}_{k G}(E(\mathcal{V})) \rightarrow \underline{\operatorname{Hom}}_{k G}(\Omega F(\mathcal{V}), k) \rightarrow H^{1}(G, k) .
$$

Proof. This is part of the long exact sequence

$$
\begin{aligned}
\ldots \rightarrow \underline{\operatorname{Hom}}_{k G}(k, k) \rightarrow \underline{\operatorname{Hom}}_{k G}(E(\mathcal{V}), k) \rightarrow \underline{\operatorname{Hom}}_{k G}(\Omega F(\mathcal{V}), k) \\
\rightarrow \underline{\operatorname{Hom}}_{k G}(\Omega k, k) \rightarrow \ldots
\end{aligned}
$$

with the second term identified by means of the lemma with $\underline{\operatorname{End}}_{k G}(E(\mathcal{V}))$.

We have $F(\mathcal{V})=\lim _{\longrightarrow} U\left(\zeta_{1}, \ldots, \zeta_{s}\right)^{*}$ as $\left(\zeta_{1}, \ldots, \zeta_{s}\right)$ runs over the sequences with $V\left(\zeta_{1}, \ldots, \zeta_{s}\right) \in \overrightarrow{\mathcal{V}}$. It follows that

$$
\begin{aligned}
\underline{\operatorname{Hom}}_{k G}(\Omega F(\mathcal{V}), k) & \cong \lim _{\longleftarrow} \underline{\operatorname{Hom}}_{k G}\left(\Omega U\left(\zeta_{1}, \ldots, \zeta_{s}\right)^{*}, k\right) \\
& \cong \widehat{\lim }^{1}\left(G, U\left(\zeta_{1}, \ldots, \zeta_{s}\right)\right) .
\end{aligned}
$$

In the special case where $\mathcal{V}$ is a finite collection of hypersurfaces (closed homogeneous subvarieties of codimension one), the direct system takes on a particularly simple form. Let $\zeta$ be an element of $H^{n}(G, k)$ whose zeros are the union $V$ of the hypersurfaces in $\mathcal{V}$, so that $V=V(\zeta)$. Then

$$
F(\mathcal{V})=F(\zeta)=\underline{\lim _{\longrightarrow}}\left(k \stackrel{\widehat{\zeta}}{\rightarrow} \Omega^{-n} k \stackrel{\widehat{\zeta}}{\rightarrow} \Omega^{-2 n} k \rightarrow \ldots\right),
$$

and so

$$
\underline{\operatorname{Hom}}_{k G}(\Omega F(\mathcal{V}), k)=\underbrace{\lim }_{t} \widehat{H}^{1-n t}(G, k) .
$$

The maps in the inverse system are given by multiplication by $\zeta$. The map from this inverse limit to $H^{1}(G, k)$ in the exact sequence of Theorem 5.5.2 is the obvious one. In particular, if there are no nonzero products in degree 1 of elements of positive degree and elements of negative degree, then the map is zero, and we have the short exact sequence

$$
0 \rightarrow k \rightarrow \underline{\operatorname{End}}_{k G}(E(\mathcal{V})) \rightarrow \underline{\operatorname{Hom}}_{k G}(\Omega F(\mathcal{V}), k) \rightarrow 0 .
$$

We can describe the product structure on $\underline{\operatorname{End}}_{k G}(E(\mathcal{V}))$ as follows. Given elements $\alpha$ and $\beta$ in

$$
\underline{\operatorname{End}}_{k G}(E(\mathcal{V})) \cong \underline{\operatorname{Hom}}_{k G}(E(\mathcal{V}), k) \cong \underbrace{\lim _{t}}_{t} \underline{\operatorname{Hom}}_{k G}\left(L_{\zeta^{t}}, k\right),
$$

write $\alpha_{t}$ and $\beta_{t}$ for their images in $\underline{\operatorname{Hom}}_{k G}\left(L_{\zeta^{t}}, k\right)$. The images $\bar{\alpha}$ and $\bar{\beta}$ of $\alpha$ and $\beta$ in $\underline{\operatorname{Hom}}_{k G}(\Omega F(\mathcal{V}), k)$ are elements of 


$$
\lim _{t} \widehat{H}^{1-n t}(G, k) \cong \lim _{t} \underline{\operatorname{Hom}}_{k G}\left(\Omega^{-n t+1} k, k\right)
$$

whose images in $H^{1}(G, k)$ are zero. Let $\bar{\alpha}_{t}$ and $\bar{\beta}_{t}$ be the images of these elements in $\underline{\operatorname{Hom}}_{k G}\left(\Omega^{-n t+1} k, k\right)$.

Of course, by passing to the sequences $\bar{\alpha}_{t}$ and $\bar{\beta}_{t}$, we have lost the multiples of the identity element in $\underline{\operatorname{End}}_{k G}(E(\mathcal{V}))$, so we only expect to be able to recover the product of $\alpha$ and $\beta$ up to linear multiples of $\alpha$ and $\beta$. This indeterminacy is precisely the indeterminacy of the Massey products appearing in the following theorem.

THEOREM 5.5.3. In the case where $\mathcal{V}$ is a finite collection of hypersurfaces with union $V=V(\zeta)$, the product $\alpha \beta$ satisfies

$$
\overline{\alpha \beta}_{t}=\left\langle\bar{\alpha}_{t}, \zeta^{t}, \bar{\beta}_{t}\right\rangle .
$$

Proof. In general, the Massey triple product $\left\langle z_{1}, z_{2}, z_{3}\right\rangle$ of elements $z_{1} \in$ $\widehat{H}^{n_{1}}(G, k), z_{2} \in \widehat{H}^{n_{2}}(G, k)$ and $z_{3} \in \widehat{H}^{n_{3}}(G, k)$ is defined provided $z_{1} z_{2}=0$ and $z_{2} z_{3}=0$, and has the following Yoneda style definition. Represent $z_{i}$ $(i=1,2,3)$ by $k G$-module homomorphisms

$$
\widehat{z}_{1}: \Omega^{n_{1}} k \rightarrow k, \quad \widehat{z}_{2}: \Omega^{n_{1}+n_{2}} k \rightarrow \Omega^{n_{1}} k, \quad \widehat{z}_{3}: \Omega^{n_{1}+n_{2}+n_{3}} k \rightarrow \Omega^{n_{1}+n_{2}} k,
$$

where $\Omega^{i+1} k$ is the $i$ th kernel in a complete (Tate) resolution

$$
\ldots \rightarrow P_{1} \rightarrow P_{0} \rightarrow P_{-1} \rightarrow \ldots
$$

of the trivial $k G$-module $k$. Since $z_{2} z_{3}=0$, the composite $\widehat{z}_{2} \widehat{z}_{3}$ factors through the projective module $P_{n_{1}+n_{2}+n_{3}-1}$. Since $k$ is simple, the fact that $\widehat{z}_{1} \widehat{z}_{2}$ factors through a projective module means it is the zero map. Therefore the composite

$$
\Omega^{n_{1}+n_{2}+n_{3}} k \rightarrow P_{n_{1}+n_{2}+n_{3}-1} \rightarrow \Omega^{n_{1}} k \rightarrow k
$$

is also the zero map, and so we get a map $\Omega^{n_{1}+n_{2}+n_{3}-1} \rightarrow k$ which represents $\left\langle z_{1}, z_{2}, z_{3}\right\rangle$. This is illustrated in the following diagram.

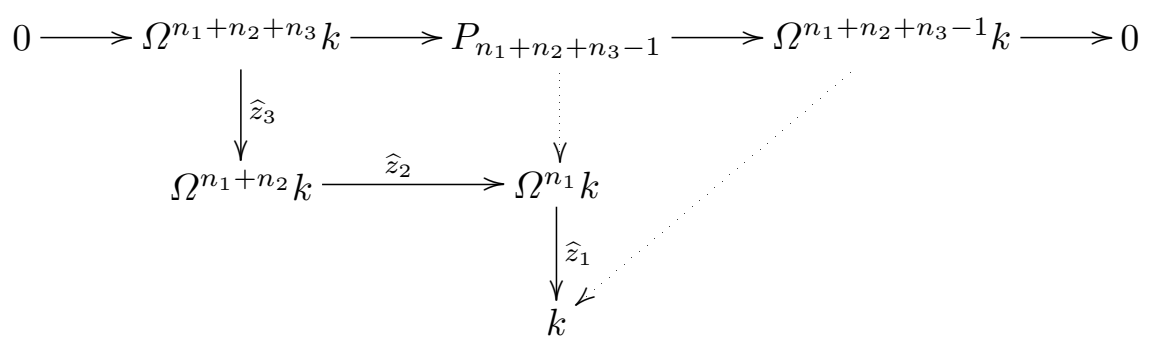

The Massey product is well defined up to the addition of multiples of $z_{1}$ and $z_{3}$. 
In the situation of the theorem, we have a diagram

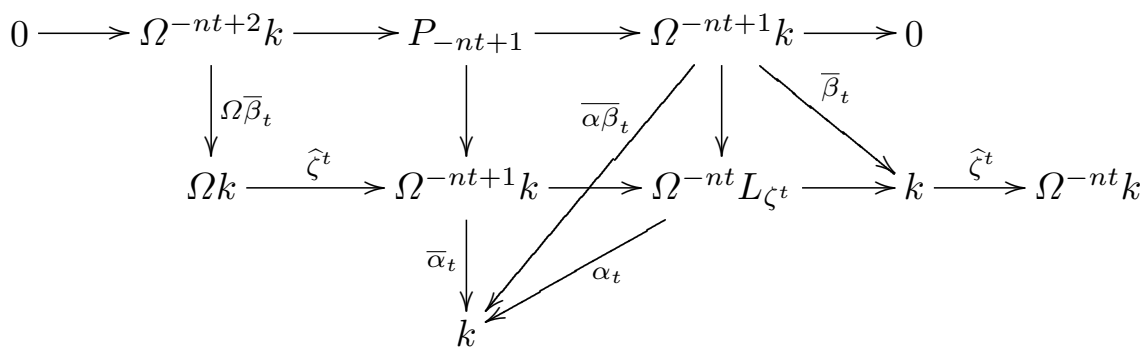

and so the Massey product is equal to $\overline{\alpha \beta}_{t}$ as required.

ExAmPLE 5.5.4. Let $G$ be the Klein four group, so that $H^{*}(G, k)=$ $k[u, v]$ is a polynomial ring in two variables of degree one. We write $\left(u^{i} v^{j}\right)^{*}$ $(i+j=n, 0 \leq i \leq n)$ for the basis elements of $\widehat{H}^{-n-1}(G, k)$ Tate dual to the basis elements $u^{i} v^{j}$ of $H^{n}(G, k)$. If we take $u$ for the element $\zeta$, then the relevant Massey products are given by

$$
\left\langle\left(u^{n-i} v^{i-1}\right)^{*}, u^{n},\left(u^{n-j} v^{j-1}\right)^{*}\right\rangle=\left(u^{n-i-j} v^{i+j-1}\right)^{*} .
$$

It follows that $\underline{\operatorname{End}}_{k G}(E(u)) \cong k[[x]]$, where $x^{i}$ is represented by $\left(u^{n-i} v^{i-1}\right)^{*}$ in $\widehat{H}^{-n}(G, k)$.

EXAMPLE 5.5.5. Let $G$ be an elementary abelian 2-group of rank $r \geq 3$, so that $H^{*}(G, k)=k\left[u_{1}, \ldots, u_{r}\right]$ is a polynomial ring on $r$ variables of degree one. In this case, if we take $u_{1}$ for the element $\zeta$, then all the Massey products involved are zero. It follows that in this case, $\operatorname{End}_{k G}\left(E\left(u_{1}\right)\right)$ is a local ring with a square zero radical of dimension $2^{\aleph_{0}}$.

\subsection{Some questions}

1. Are the idempotent modules $E(V)$ constructed by Rickard [30] always pure injective? We have only proved this in the special case where $G$ has $p$-rank two (Theorem 5.3.1). If $\mathcal{U}$ denotes the set of all proper closed homogeneous irreducible subvarieties of $V_{G}$, is the idempotent module $F(\mathcal{U})$ always pure injective? We have only proved this in the special case where $G$ is an elementary abelian group of rank two (Theorem 5.4.6).

2. What is the pure global dimension of the group algebra of the dihedral groups of order at least eight, the semidihedral groups and the generalized quaternion groups? These are the cases left open in Theorem 4.5.3. There should also be a blockwise version of this theorem, but it seems harder to get at.

3. Given a finite group $G$ and a prime $p$ dividing $|G|$, does there exist a number $n_{p}(G)$ such that given any field $k$ of characteristic $p$, the composite 
of $n_{p}(G)$ phantom maps between $k G$-modules factors through a projective module? We proved this in Section 4.6 in case $p=2$ and $G$ has 2-rank two.

\section{References}

[1] F. W. Anderson and K. R. Fuller, Rings and Categories of Modules, Grad. Texts in Math. 13, Springer, Berlin, 1974.

[2] M. Auslander, Representation theory of Artin algebras II, Comm. Algebra 1 (1974), 269-310.

[3] D. Baer, H. Brune and H. Lenzing, A homological approach to representations of algebras II: tame hereditary algebras, J. Pure Appl. Algebra 26 (1982), 141-153.

[4] D. Baer and H. Lenzing, A homological approach to representations of algebras I: the wild case, ibid. 24 (1982), 227-233.

[5] D. J. Benson, Representations and Cohomology I: Basic representation theory of finite groups and associative algebras, Cambridge Stud. Adv. Math. 30, Cambridge Univ. Press, 1991.

[6] -, Representations and Cohomology II: Cohomology of groups and modules, Cambridge Stud. Adv. Math. 31, Cambridge Univ. Press, 1991.

[7] —, Cohomology of modules in the principal block of a finite group, New York J. Math. 1 (1995), 196-205.

[8] D. J. Benson and J. F. Carlson, Products in negative cohomology, J. Pure Appl. Algebra 82 (1992), 107-129.

[9] D. J. Benson, J. F. Carlson and J. Rickard, Complexity and varieties for infinitely generated modules, I, Math. Proc. Cambridge Philos. Soc. 118 (1995), 223243.

[10] - - - - Complexity and varieties for infinitely generated modules, II, ibid. 120 (1996), 597-615.

[11] - - - - , Thick subcategories of the stable module category, Fund. Math. 153 (1997), 59-80.

[12] J. F. Carlson, P. W. Donovan and W. W. Wheeler, Complexity and quotient categories for group algebras, J. Pure Appl. Algebra 93 (1994), 147-167.

[13] J. F. Carlson and W. W. Wheeler, Homomorphisms in higher complexity quotient categories, to appear.

[14] J. D. Christensen, Ideals in triangulated categories: phantoms, ghosts and skeleta, Adv. Math. 136 (1998), 284-339.

[15] J. D. Christensen and N. P. Strickland, Phantom maps and homology theories, Topology 37 (1998), 339-364.

[16] G. Ph. Gnacadja, Phantom maps in the stable module category, J. Algebra 201 (1998), 686-702.

[17] B. Gray, Spaces of the same n-type, for all n, Topology 5 (1966), 241-243.

[18] L. Gruson et C. U. Jensen, Modules algébriquement compacts et foncteurs $\varliminf^{(i)}$, C. R. Acad. Sci. Paris Sér. A-B 276 (1973), A1651-A1653.

[19] - - - Dimensions cohomologiques reliées aux foncteurs $\varliminf^{(i)}$, in: Séminaire d'Algèbre Paul Dubreil et Marie-Paule Malliavin, Lecture Notes in Math. 867, Springer, Berlin, 1981, 234-294.

[20] A. Heller, The loop-space functor in homological algebra, Trans. Amer. Math. Soc. 96 (1960), 382-394. 
[21] M. Hovey, J. H. Palmieri and N. P. Strickland, Axiomatic stable homotopy theory, Mem. Amer. Math. Soc. 610 (1997).

[22] C. U. Jensen and H. Lenzing, Model Theoretic Algebra, Gordon and Breach, 1989.

[23] H. Krause, Generic modules over Artin algebras, Proc. London Math. Soc. (3) 76 (1998), 276-306.

[24] J. Lambek, Lectures on Rings and Modules, Blaisdell, Waltham, MA, 1966.

[25] C. A. McGibbon, Phantom maps, in: Handbook of Algebraic Topology, I. M. James (ed.), North-Holland, Amsterdam, 1995, 1209-1257.

[26] A. Neeman, On a theorem of Brown and Adams, Topology 36 (1997), 619-645.

[27] B. L. Osofsky, Homological dimension and the continuum hypothesis, Trans. Amer. Math. Soc. 132 (1968), 217-230.

[28] —, Homological Dimensions of Modules, CBMS Regional Conf. Series Math. 12, Amer. Math. Soc., 1973.

[29] M. Prest, Model Theory and Modules, London Math. Soc. Lecture Note Ser. 130, Cambridge Univ. Press, 1988.

[30] J. Rickard, Idempotent modules in the stable category, J. London Math. Soc. 178 (1997), 149-170.

[31] J. E. Roos, Sur les foncteurs dérivés de lim. Applications, C. R. Acad. Sci. Paris 252 (1961), 3702-3704.

[32] R. B. Warfield, Purity and algebraic compactness for modules, Pacific J. Math. 28 (1969), 699-719.

[33] A. Zabrodsky, On phantom maps and a theorem of H. Miller, Israel J. Math. 58 (1987), 129-143.

[34] M. Ziegler, Model theory of modules, Ann. Pure Appl. Logic 26 (1984), 149-213.

Department of Mathematics

University of Georgia

Athens, GA 30602

U.S.A.

E-mail: djb@byrd.math.uga.edu gnacadja@math.uga.edu 